\title{
Binary black hole late inspiral: Simulations for gravitational wave observations
}

\author{
John G. Baker, ${ }^{1}$ Sean T. McWilliams, ${ }^{2}$ James R. van Meter, ${ }^{1,3}$ Joan Centrella, ${ }^{1}$ Dae-Il Choi, ${ }^{1,4,5}$ \\ Bernard J. Kelly, ${ }^{1}$ and Michael Koppitz ${ }^{1,6}$ \\ ${ }^{1}$ Gravitational Astrophysics Laboratory, NASA Goddard Space Flight Center, 8800 Greenbelt Rd., Greenbelt, Maryland 20771, USA \\ ${ }^{2}$ University of Maryland, Department of Physics, College Park, Maryland 20742, USA \\ ${ }^{3}$ Center for Space Science \& Technology, University of Maryland Baltimore County, Physics Department, 1000 Hilltop Circle, \\ Baltimore, Maryland 21250, USA \\ ${ }^{4}$ Universities Space Research Association, 10211 Wincopin Circle, Suite 500, Columbia, Maryland 21044, USA \\ ${ }^{5}$ Korea Institute of Science and Technology Information, 52-11, Eoun-Dong, Yuseong-Gu, Daejeon, South Korea, $305-806$ \\ ${ }^{6}$ Albert Einstein Institute, Am Mühlenberg 1, 14471 Golm, Germany
}

(Received 19 December 2006; revised manuscript received 20 April 2007; published 28 June 2007)

\begin{abstract}
Coalescing binary black hole mergers are expected to be the strongest gravitational wave sources for ground-based interferometers, such as the LIGO, VIRGO, and GEO600, as well as the space-based interferometer LISA. Until recently it has been impossible to reliably derive the predictions of general relativity for the final merger stage, which takes place in the strong-field regime. Recent progress in numerical relativity simulations is, however, revolutionizing our understanding of these systems. We examine here the specific case of merging equal-mass Schwarzschild black holes in detail, presenting new simulations in which the black holes start in the late-inspiral stage on orbits with very low eccentricity and evolve for $\sim 1200 M$ through $\sim 7$ orbits before merging. We study the accuracy and consistency of our simulations and the resulting gravitational waveforms, which encompass $\sim 14$ cycle before merger, and highlight the importance of using frequency (rather than time) to set the physical reference when comparing models. Matching our results to post-Newtonian (PN) calculations for the earlier parts of the inspiral provides a combined waveform with less than one cycle of accumulated phase error through the entire coalescence. Using this waveform, we calculate signal-to-noise ratios (SNRs) for iLIGO, adLIGO, and LISA, highlighting the contributions from the late-inspiral and merger-ringdown parts of the waveform, which can now be simulated numerically. Contour plots of SNR as a function of $z$ and $M$ show that adLIGO can achieve SNR $\gtrsim 10$ for some intermediate mass binary black holes (IMBBHs) out to $z \sim 1$, and that LISA can see massive binary black holes (MBBHs) in the range $3 \times 10^{4} \lesssim M / M_{\odot} \lesssim 10^{7}$ at SNR $>100$ out to the earliest epochs of structure formation at $z>15$.
\end{abstract}

DOI: 10.1103/PhysRevD.75.124024

PACS numbers: 04.25.Dm, 04.30.Db, 04.70.Bw, 04.80.Nn

\section{INTRODUCTION}

Coalescing binary black holes (BBHs) are strong sources of gravitational radiation for ground-based detectors such as LIGO, VIRGO, and GEO600, and for the space-based LISA. This coalescence is generally considered to proceed in three phases: inspiral, merger, and ringdown [1]. During the inspiral stage, the black holes are well separated and spiral together on quasicircular orbits. This is followed by the merger stage, during which the black holes plunge towards the center and merge together, forming a common horizon. This distorted remnant then "rings down" to form a Kerr black hole by shedding its nonaxisymmetric modes as gravitational radiation.

Different techniques are used to calculate the gravitational radiation from these stages. The inspiral can be treated analytically with post-Newtonian (PN) techniques; the gravitational waveform is a chirp, a sinusoid that increases in both amplitude and frequency. The later part of the ringdown can be handled analytically using black hole perturbation theory, with the resulting waveforms being exponentially damped sinusoids of constant fre- quency. However, calculating the waveforms from the dynamical merger, which produces the highest luminosity signal, requires numerical relativity simulations of the full Einstein equations in three spatial dimensions plus time. With the first generation of ground-based detectors now taking data and LISA moving forward, knowledge of the merger waveforms and their impact on detectability and parameter estimation is urgent.

Recently there has been dramatic progress in numerical relativity calculations of BBH mergers. The first full orbit of an equal-mass nonspinning BBH was achieved nearly three years ago [2] (see also [3]), using a conformal formalism and comoving coordinates, with the black holes represented as punctures [4], and held fixed in the grid. This was followed about a year and a half later by the first simulation of the final orbit, merger, and ringdown [5]; this work was carried out using generalized harmonic coordinates with excised black holes moving through the computational domain [6,7]. Roughly six months later the moving puncture method, which is based on a conformal formalism and allows the puncture black holes to move across the grid without the need for excision $[8,9]$, was 
introduced. This simple yet powerful technique proved to be highly effective [10-13], allowing the evolution of black holes through multiple orbits, merger, and ringdown $[14,15]$. Simulations with unequal masses [16-18] and with spins $[19,20]$ quickly followed. Longer simulations with several orbits before merger have also been carried out using excision and harmonic techniques [21], allowing comparisons between the resulting waveforms [22].

This explosion of new work on BBH mergers has opened up exciting opportunities for applications of these results. A number of key applications center on gravitational wave data analysis. In particular, the need for accurate templates for the full gravitational wave signalencompassing the inspiral, merger, and ringdown phases - means it is essential to understand the relationship between PN waveforms and the results from numerical relativity $[15,21,23]$. And, with longer simulations now becoming available, it is becoming feasible to apply these waveforms to questions about the detectability of BBHs for various detectors [21]. In this paper, we focus especially on issues of accuracy in long simulations, starting in the lateinspiral regime, and their applications in gravitational wave data analysis.

The most rapid advances cover the previously least understood portion of the radiation, the final few cycles of radiation generated from near the "innermost stable circular orbit" (ISCO) and afterward, which we call the "merger-ringdown." Already there has been considerable progress toward a full understanding of this important portion of the waveform through which the frequency sweeps by a factor of $\sim 3$ up to ringdown. A significant development in this regard was the demonstration of initial-data independence of merger waveforms for equalmass, spinless black holes [15]. Today, there is general agreement that the merger of such a system produces a final Kerr BH remnant with spin $a / m \sim 0.7$, and that the amount of energy radiated in the form of gravitational waves, starting with the final few orbits and proceeding through the plunge, merger, and ringdown, is $E_{\mathrm{rad}} \sim 4 \%$ $[5,8,9,14,15]$; see [24] for a review. There is also a consensus on the overall simple shape of the waveforms; a detailed comparison of results among numerical relativity groups has already begun [22], with more to follow. The exploration of parameter space, including various mass ratios [16-18] and spins [19,20], is now underway. Future efforts will involve pushing this frontier to increasingly complex mass-ratio and spin-orientation combinations, and to establishing initial-data independence across these parameters.

Though more challenging, it is now becoming practical to simulate BBHs starting in the late inspiral as well. We report on new simulations covering roughly an additional factor of 3 in frequency before the "merger-ringdown." We have simulated the coalescence of equal-mass, nonspinning black holes, starting in the late-inspiral regime, and continuing through the merger and ringdown. The black holes start on orbits with very low eccentricity and spiral together through $\sim 7$ orbits before merging. The resulting gravitational waveform has $\sim 14$ cycle, enabling a robust consistency test with late-inspiral PN waveforms as reported in [23]. In Sec. II we present an overview of our numerical results. Readers with an interest in the details of the simulations will find them in Sec. III, where we discuss our methodology and the accuracy of our simulations, as well as introduce the important notion of considering physical quantities as functions of frequency rather than time when comparing predictions from different computations.

The rapid advances in understanding merger-ringdown waveforms with emerging simulation results for the late inspiral create a new context for considering gravitational wave observations of this part of the signal from such a system. In Sec. IV we revisit an analysis of merger detectability originally carried out in Flanagan and Hughes [1], now in the context of presentday and emerging waveform modeling capabilities. In particular, we examine the relative contributions to the signal-to-noise ratio (SNR) for the merger-ringdown and late-inspiral portions of equal-mass coalescence signals, as observed by initial LIGO (iLIGO), advanced LIGO (adLIGO), or LISA. We also plot contours of SNR as functions of redshift $z$ and total mass for adLIGO and LISA, showing their ability to detect BBHs in the cosmos. These SNR calculations draw on a refined waveform model based on a best-estimate waveform using both numerical relativity and PN results. We conclude in Sec. V with a summary and discussion of our main results.

\section{OVERVIEW OF SIMULATION RESULTS}

Late-inspiral evolutions, covering more than a few orbits prior to ringdown, are more challenging than shorter merger-ringdown simulations. In this regime there is a stronger requirement for numerical stability and a greater demand for computational resources. In addition, better accuracy is required to control the accumulated phase error, which in turn constrains the numerical error that can be tolerated in the rate of energy loss.

Using the moving puncture technique [8-10], with the gauge evolution given by Eq. (17) and Eq. (26) in [10], we have simulated the evolution of a nonspinning equal-mass $\mathrm{BBH}$ starting at relatively wide separation, $\sim 1200 \mathrm{M}$ or $\sim 7$ orbits before the formation of a common event horizon. Here $M$ is the total mass the system would have had when the black holes were very far apart and before radiative losses became significant. We used fourth-order-accurate finite differencing techniques with adaptive mesh refinement (AMR) to resolve the dynamics near the black holes and in the wave propagation region. We carried out three runs using similar grid refinement structures, but at different resolutions: low $\left(h_{f}=3 M / 64\right)$, medium $\left(h_{f}=\right.$ $3 M / 80)$, and high $\left(h_{f}=M / 32\right)$. Here, $h_{f}$ is the grid 


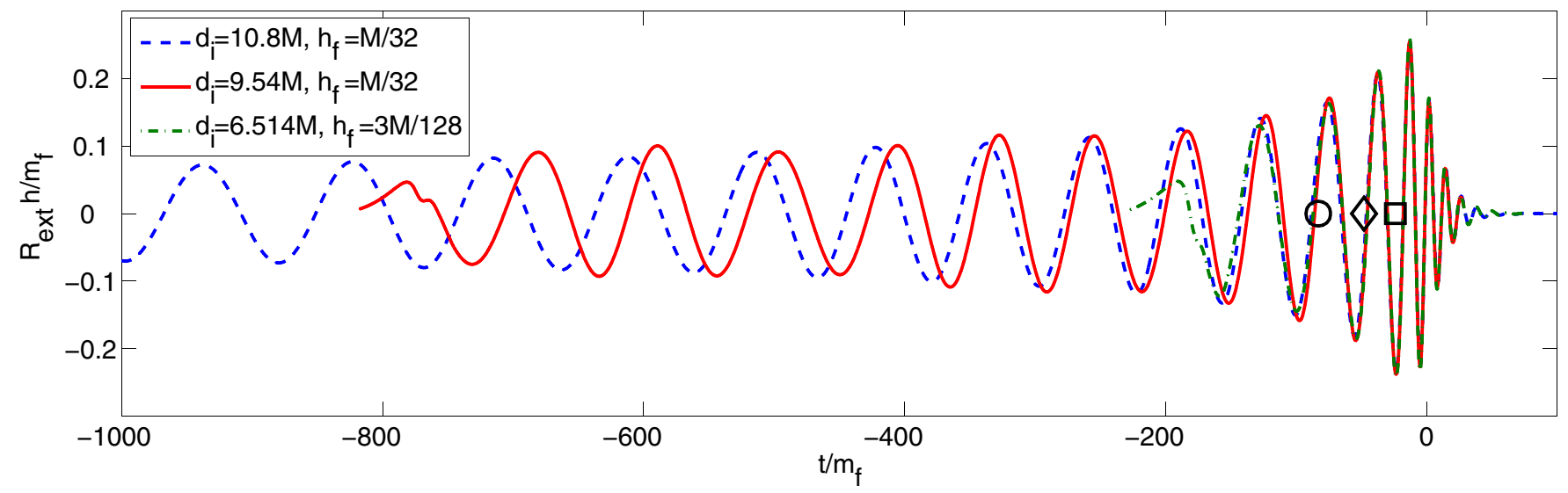

FIG. 1 (color online). Gravitational strain waveforms from the merger of equal-mass Schwarzschild black holes. The late part of the merger $(t \geq-50 M)$ is robustly determined and relatively easily calculable, while simulations of the late inspiral (early part of the waveform) are rapidly approaching the phasing accuracy required for observational applications [23]. The solid line shows our current "best" numerical waveform. The dashed line shows a comparison waveform from a run starting with the same initial data as R4 in Ref. [15] and the dash-dotted curve shows the results from the highest resolution R1 run in Ref. [15]. All waveforms have been extracted at $R_{\text {ext }}=40 M$ and shifted in time so that the moment of maximum $\psi_{4}$ radiation amplitude occurs at time $t=0$. The initial coordinate distance between the punctures, $d_{i}$, is indicated in all cases.

spacing in the regions with the highest resolution in each simulation, those being the regions around each black hole. Overall, the Hamiltonian constraint converges at fourth order, and the momentum constraint at better than second order, throughout the runs.

Figure 1 shows the resulting gravitational waveform in the context of recent developments in black hole evolutions. The dashed curve gives the gravitational wave strain from the dominant $l=2, m=2$ spin-weighted spherical harmonic from our highest resolution simulation, extracted at $R_{\text {ext }}=40 M$. This represents an observation made on the equatorial plane of the system, where only a single polarization component contributes to the measured strain. Time $t=0$ is taken to be the moment of peak radiation amplitude as measured by the Weyl curvature $\psi_{4}$; the symbols along the time axis mark the points at which the system reaches the ISCO calculated for a Schwarzschild black hole (circle), EOB 3PN [25,26] (diamond), and adiabatic 3 PN $[26,27]$ (square). For comparison, we show two other waveforms from earlier simulations carried out by this group; both were extracted at $R_{\text {ext }}=40 M$ and have been shifted in time and initial phase so (as in [15]) that the moment of peak $\psi_{4}$ radiation amplitude occurs at $t=0$. As these different simulations may radiate different fractions of the initial mass, we choose the mass $m_{f}$ of the postmerger Kerr hole as the natural length scale for comparison (see discussion of Table I for details). Because of radiative losses, $m_{f} \approx 0.95 M$.

The solid curve shows the results from a model that starts $\sim 550 M$ before merger with the same initial data as run R4 in Ref. [15] but using a different gauge. Note that the gauge conditions used for the dashed curve and the solid curve are equivalent to those given by case \#8 in Ref. [10], while the gauge conditions used in Ref. [15] are equivalent to those given by case \#3 in Ref. [10]. The dotdash curve shows a simulation that starts $\sim 200 M$ before merger; this is the high resolution run R1 from Ref. [15]. All three waveforms agree to within $\sim 1 \%$ for the mergerringdown burst part of the waveform, starting near $t \sim$ $-50 M$.

Astrophysically, BBHs in this relatively late stage of their evolution are expected to be traveling on nearly circular orbits, as any initial eccentricity would have been radiated away early in their evolution. In this new model, we start the black holes on nearly circular orbits with very small eccentricity $e<0.01$, as defined below. The resulting black hole trajectories are shown in Fig. 2, where the tracks mark the paths of the punctures; for

TABLE I. The parameters of the final black hole (mass and spin parameter) formed by merger. Here $m_{r}$ and $(a / m)_{r}$ are calculated from the complex ringdown frequency $\omega_{r}$, while $m_{f}$ and $(a / m)_{f}$ are calculated from the radiation measured at the given radius. Analysis of these quantities at $R_{\text {ext }}=40 M$ across the three different resolutions suggests that they are at least second-order convergent, and comparison across extraction radii gives error bars for the $h_{f}=M / 32$ results for $m_{r},(a / m)_{r}, m_{f}$, and $(a / m)_{f}$ of $1 \%, 1 \%, 0.3 \%$, and $3 \%$, respectively.

\begin{tabular}{cc|ccccc}
\hline \hline & & \multicolumn{3}{|c}{ Ringdown } & \multicolumn{3}{c}{ Conservation } \\
$h_{f}$ & $R_{\text {ext }}$ & $\omega_{r}$ & $m_{r}$ & $(a / m)_{r}$ & $m_{f}$ & $(a / m)_{f}$ \\
\hline $3 M / 64$ & $40 M$ & $0.551-0.083 i$ & 0.971 & 0.71 & 0.953 & 0.70 \\
$3 M / 80$ & $40 M$ & $0.553-0.087 i$ & 0.945 & 0.68 & 0.954 & 0.72 \\
& $60 M$ & $0.553-0.088 i$ & 0.931 & 0.66 & 0.955 & 0.70 \\
& $100 M$ & $0.553-0.088 i$ & 0.931 & 0.66 & 0.959 & 0.71 \\
$M / 32$ & $40 M$ & $0.553-0.085 i$ & 0.953 & 0.69 & 0.955 & 0.73 \\
& $60 M$ & $0.553-0.085 i$ & 0.953 & 0.69 & 0.955 & 0.71 \\
& $100 M$ & $0.553-0.086 i$ & 0.945 & 0.68 & 0.958 & 0.71 \\
\hline \hline
\end{tabular}




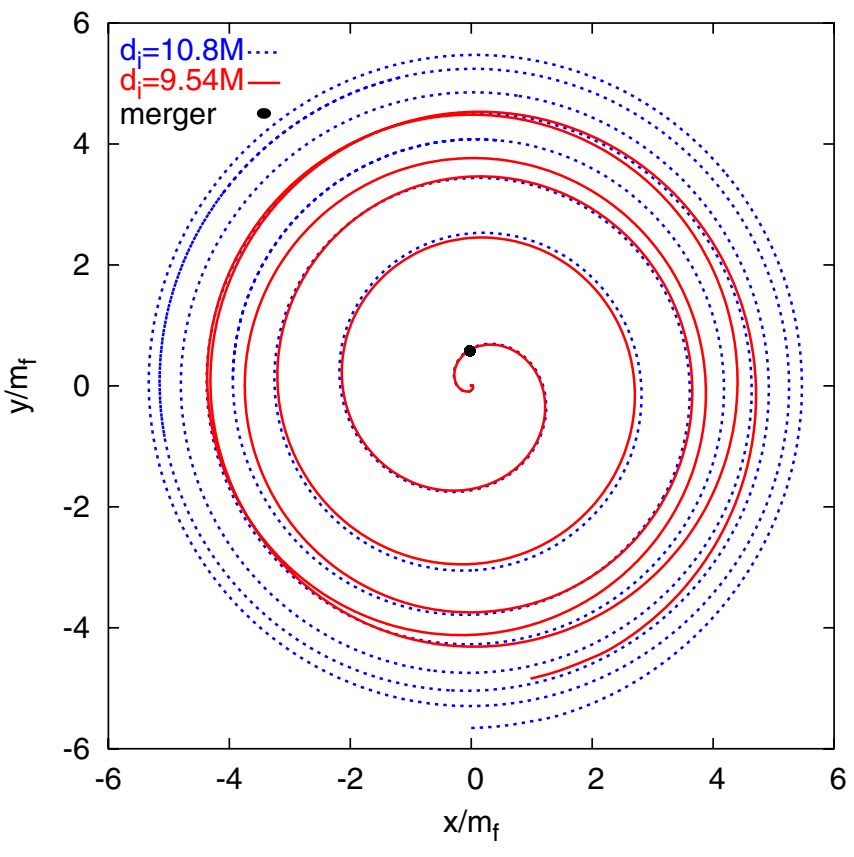

FIG. 2 (color online). The trajectory of one of the binary system's black holes through $\sim 7$ revolutions before coalescence for our high-resolution case is shown by the dotted line. The solid line gives the trajectory of the moderately long comparison run. The initial coordinate distance between the punctures, $d_{i}$, is indicated in both cases.

clarity, only a single black hole from each simulation is shown. The dashed curve shows the results from our new high resolution long run; note that the early orbits are very nearly circular. The solid curve shows the trajectory of the moderately long comparison run (shown by the dashed line in Fig. 1). At early times, this model is significantly more eccentric than our new results. At later times this trajectory locks on to that of our new run, $\sim 2.5$ orbits before merger.

The black hole separation as a function of time is shown for these two models in Fig. 3. The greater eccentricity of the previous model (solid curve) is clearly distinguished here. We also show all three resolutions of our newest model. The slight deviations from the overall smooth trend give an indication of the small amount of eccentricity in these latter simulations. Note however the differences between these three resolutions, particularly in the total time between the start of the simulation and the merger. Although significant, the differences in merger time appear to converge at a rate consistent with fourth-order error.

In the next section we consider numerical techniques and the fidelity of the simulations in more detail, including the convergence and conservation properties. We also carry out a detailed analysis of the resulting gravitational waveforms, underscoring the differences between using time and frequency to set references for comparing models. Readers more interested in issues of detectability and SNR analyses for iLIGO, adLIGO, and LISA are invited to skip to Sec. IV.

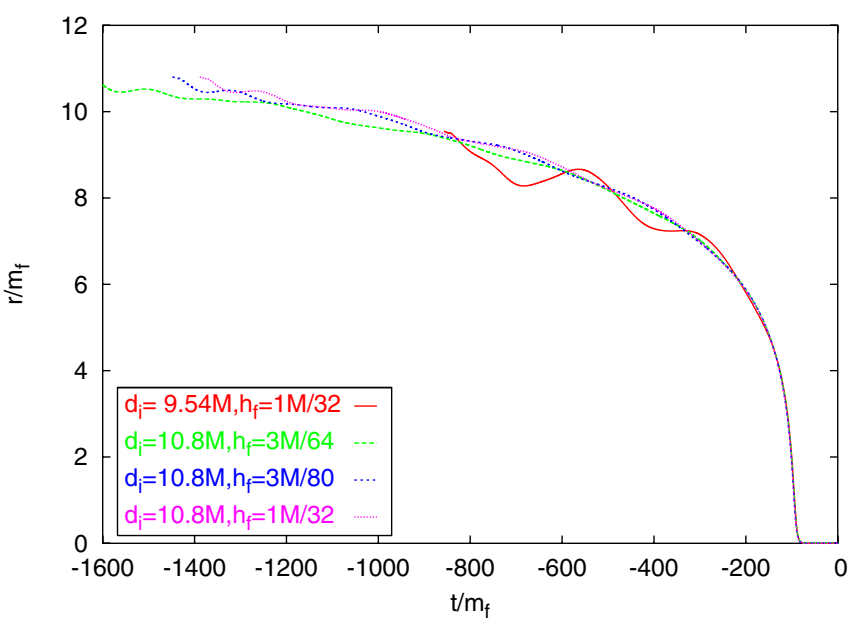

FIG. 3 (color online). The coordinate separation between the puncture black holes is shown as a function of time. The solid line shows the results for the comparison run, which has relatively large eccentricity. The other curves show the three resolutions for our new simulations, all having noticeably less eccentricity. Note that equivalent gauge evolution equations were used in all four cases.

\section{SIMULATION DETAILS}

\section{A. Numerical methodology}

For initial data we used Brandt-Brügmann puncture data [4], generated by a second-order-accurate multigrid solver, AMRMG [28]. The puncture parameters were determined by the Tichy-Brügmann prescription for quasicircular initial data [29], adjusted slightly, as informed by previous empirical experience, to reduce eccentricity. Our adjustment was simply to reduce the initial coordinate separation by roughly $2 \%$ while increasing the initial momentum of each puncture such that the product of the initial momentum with the initial coordinate separation remains constant. The success of this approach in giving a circular inspiral is roughly indicated by the puncture track (dotted line) in Fig. 2 and the curves in Fig. 3. The puncture track was computed by integrating the equation $\dot{\vec{x}}_{\text {punc }}=-\vec{\beta}\left(\vec{x}_{\text {punc }}\right)$, where $x_{\text {punc }}$ is the position of the puncture, and $\vec{\beta}$ the shift.

This data was evolved using standard BaumgarteShapiro-Shibata-Nakamura (BSSN) evolution equations, with the addition of dissipation terms as in [30] and constraint-damping terms as in [31] in order to ensure robust stability. The dissipation terms, however, were tapered with Gaussian functions so as to vanish at each puncture position; this modification proved necessary for accuracy. The gauge condition was that recommended in [10] for moving punctures. Time integration was performed with a fourth-order Runge-Kutta algorithm, and spatial derivatives with fourth-order-accurate finitedifferencing stencils. For the outer boundary we employed a second-order-accurate Sommerfeld condition, pushed to 
$\left|x_{i}\right|=1536 M$ to keep reflections far from the source. AMR was implemented via the software package PARAMESH [32,33], and interpolation between refinement regions was fifth-order-accurate. Note that we use AMR only to resolve the sources, and the mesh will progressively become coarser far away from the sources. Although the radiation which reaches the outer boundary during the course of the simulation, with wavelengths of $\gtrsim 100 M$, will not be well resolved in this lowest refinement region of grid spacing $h=32 M$ (in the highest resolution run), reflections from there are causally disconnected from our extraction radii at $R \leq 100 M$. We do not use AMR to follow the radiation with fine mesh; instead we require only that the fixed mesh resolution in the region of the extraction surfaces be sufficient to resolve the waves there. For example the extraction surface at $R=60 M$, in our highest resolution simulation, spans regions with grid spacing $h=1 M$ and $h=2 M$.

\section{B. Simulation analysis}

The accuracy of the simulations was assessed by various means. We first considered the $L_{1}$ norm of the constraints in each refinement region, the grid structure having been designed to be commensurate for all resolutions; results for the finest (top panel) and second finest (bottom panel) refinement regions are plotted in Figs. 4 and 5. The Hamiltonian and momentum constraints were found to be convergent at an apparent order of 2.5 in the finest grid, where the error at the puncture can be expected to dominate and fourth-order finite differencing must break down due to the irregularity there. In all coarser regions the
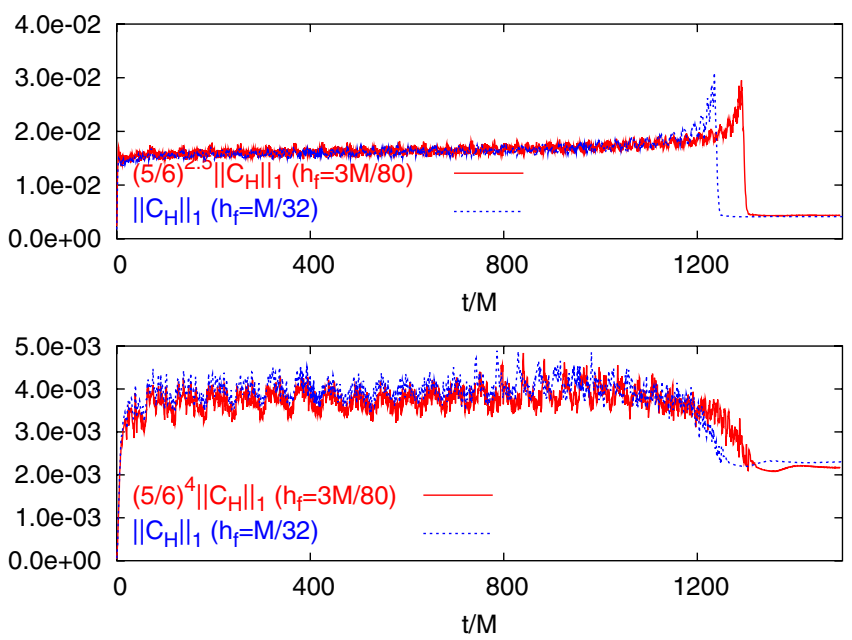

FIG. 4 (color online). Convergence plot for the Hamiltonian constraint $C_{H}$. The top panel shows results from the finest grid and has been scaled so that for 2.5 order convergence the curves should superpose. The bottom panel shows results from the second finest grid and has been scaled so that for fourth-order convergence the curves should superpose; the curves indeed appear to be fourth-order convergent.
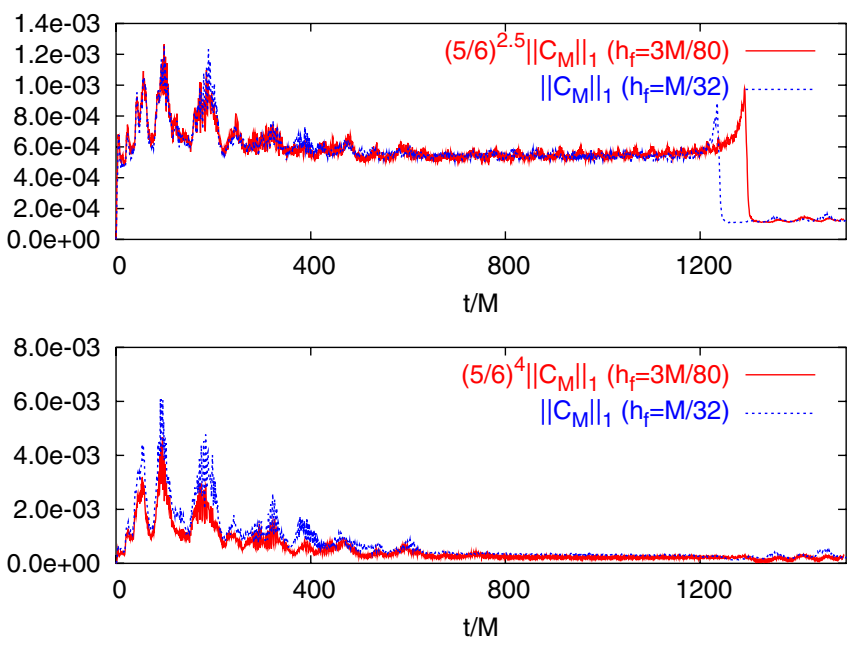

FIG. 5 (color online). Convergence plot for the momentum constraint $C_{M}$. The top panel shows results from the finest grid and has been scaled so that for 2.5 order convergence the curves should superpose. The bottom panel shows results from the second finest grid and has been scaled so that for fourth-order convergence the curves should superpose; the curves appear less than fourth-order convergent but better than second-order convergent.

Hamiltonian constraint appears to be fourth-orderconvergent, while the momentum constraint appears closer to second-order convergent.

From each simulation we have measured the radiation in the form of the complex Weyl tensor component $\psi_{4}$, specified consistently with [34] to leading order in $1 / r$. The gravitational wave strain is related to $\psi_{4}$ by $-\ddot{h}_{+}+i \ddot{h}_{\times}=$ $2 \psi_{4}$, and can be recovered by integration, with the two complex integration constants chosen to keep the strain close to oscillating about zero. For some applications we also examine waveforms in the form of the strain rate $v=$ $\dot{h}_{+}+i \dot{h}_{\times}$, the quantity from which radiation energy is directly obtained. To extract the waveform information from the simulation we define a series of coordinate spheres of different radii $R_{\text {ext }} / M \in\{40,60,100\}$ on which we measure spin-weighted spherical harmonic components of $\psi_{4}$ via a second-order algorithm described in [35,36]. In this paper we focus exclusively on the $l=2, m=2$ component of the radiation, which mirrors the $l=2, m=-2$ component because of equatorial symmetry. Other components are considerably smaller, containing $\sim 1 \%$ as much energy [15].

Figure 6 compares waveforms from our high-resolution simulation extracted on each of our three extraction spheres with the $R_{\text {ext }}=40 M$ and $R_{\text {ext }}=60 M$ waveforms shifted in time by the intervening propagation time derived based on a Schwarzschild black hole background. The generally good agreement for all three waveforms indicates that potentially worrying subtleties related to the relatively close distance of the extraction spheres, such as nonlinear propagation effects, or tetrad-specification sen- 


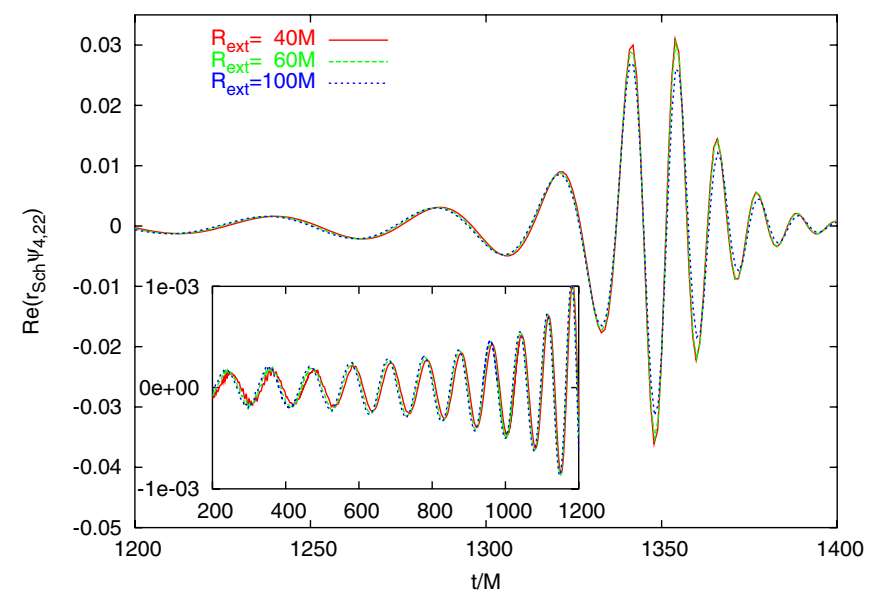

FIG. 6 (color online). $\quad \psi_{4}$ waveform calculated at three different extraction radii, and scaled by the approximate Schwarzschild areal radius. Times have been shifted according to the approximate Schwarzschild "tortoise coordinate" [35,53], as appropriate to compare the $R_{\text {ext }}=40 M$ and $R_{\text {ext }}=60 M$ waveforms with the $R_{\text {ext }}=100 M$ waveform.

sitivity, do not seriously affect the waveforms. On the other hand, some small differences are evident. For the early portion of the waveforms, shown in the inset, the results from the closest extraction sphere show slight differences in amplitude and phase, suggesting that $1 / R_{\text {ext }}^{2}$ radiation details are not yet insignificant here; this is not surprising given that the extraction radius in this case is only $\sim 1 / 4$ of a wavelength. On the other hand, the waveform from the farthest extraction radius shows signs of dissipation for the later higher-frequency portion of the waveform. This is because the radiation has propagated significantly farther, through a lower-resolution region on the computational grid, by the time it is measured at $R_{\text {ext }}=100 M$. The resolution in most of the intermediate region is $h=2 M$, about six points per cycle for the ringdown radiation. For the rest of the paper, we primarily employ the waveforms extracted at the intermediate distance $R_{\text {ext }}=60 M$, which is only weakly affected by either of the above short- and long-wavelength effects.

Different information in the waveforms provides independent ways to deduce the energy and angular momentum of the final black hole produced by the merger. The ringdown dynamics of the black hole after merger contains direct information about the mass and spin parameter of the merged black hole, so that we can deduce $m_{r}$ and $(a / m)_{r}$ by measuring the frequency and decay rate of the ringdown radiation. Alternatively, we can measure the radiation energy $E_{\text {rad }}$ and angular momentum content $J_{\text {rad }}$ at a given radius. Then, since we know the initial values, conservation of energy and angular momentum imply the values of $m_{f}=\left.M_{\mathrm{ADM}}\right|_{t=0}-E_{\mathrm{rad}}$ and $(a / m)_{f}=\left(\left.J_{\mathrm{ADM}}\right|_{t=0}-\right.$ $\left.J_{\text {rad }}\right) / m_{f}^{2}$. Results from both methods are shown in Table I. By comparing $m_{f}$ with $m_{r}$ and $(a / m)_{r}$ with $(a / m)_{f}$, we can verify conservation of energy and angular momentum in the simulations. In Table I we see that at the highest resolution, energy is conserved to within $\sim 1 \%$ and angular momentum to within $\sim 6 \%$; and the best conservation is seen at $R_{\text {ext }}=60 M$, where energy is conserved to within $\sim 0.2 \%$ and angular momentum to within $\sim 3 \%$.

To an excellent approximation, the $l=2, m=2$ harmonic of the radiation is polarized in the form expected for radiation generated by circular motion. The polar representation of the $l=2, m=2$ component of the complex waveform, $\psi_{4,22}=A_{\psi}(t) \exp \left(i \phi_{\psi}(t)\right)$, is particularly natural for circularly polarized radiation, for which $A_{\psi}$ and $\omega=\partial \phi_{\psi} / \partial t$ vary only slowly. The angular polarization frequency $\omega$ then provides a meaningful instantaneous frequency obtained directly from the radiation, corresponding to twice the angular frequency of orbital motion when the black holes are still separate. Because the radiation is measured in the weak-field region of our simulations, where gauge dependence is minimal, this polarization frequency provides gauge-invariant information about the binary dynamics.

If the orbital motion is eccentric, this will leave an imprint in the radiation, causing a slight decrease in the polarization frequency of radiation generated near apocenter. We can recognize eccentric motion by identifying periodic deviations from a smooth monotonic trend in the time development of the polarization frequency $\omega(t)$.

Specifically, we looked at the polarization frequency $\omega(t)=\partial \phi / \partial t$ calculated from the strain rate $v(t)=$ $V(t) \exp (i \phi(t))$. We see generally similar results whether we use strain, strain rate, or $\psi_{4}$ to define the frequency, but specifically show the strain rate because it comes out smoother than $\psi_{4}$ with respect to small waveform noise, but without noticeable detrending issues as in the strain. We fit the time dependence of the frequency curves to a quartic trend, $\omega_{c}$, plus an eccentric modulation of the form $d \omega=A \sin \left(\Phi_{0}+\Omega_{0}\left(t-t_{0}\right)+\dot{\Omega}\left(t-t_{0}\right)^{2}\right)$ where the quantities $A, \Phi_{0}, \Omega_{0}$, and $\dot{\Omega}$ are fitting constants and $t_{0}=61 \mathrm{M}$ is a time offset approximately accounting for the propagation time to $R_{\mathrm{ext}}=60 \mathrm{M}$. Ignoring the early part of the simulation where there are transient initial-data effects, and the late part where the secular trend is very strong, we fit the curves over the time range $250 M<t<1000 M$. We get similar results for eccentricity whether we apply highfrequency filtering to the waveform before fitting, though we show only low-pass filtered curves in Fig. 7. The results of the fitting are summarized in Table II.

Variations in the details of the fitting procedure, such as using strain instead of strain rate or smoothing highfrequency noise from $\omega(t)$, give results consistent to roughly the number of significant digits shown for $A$ and $\Omega_{0}$, though $\dot{\Omega}$ and $\Phi_{0}$ vary more significantly in some cases. The period of eccentric oscillations indicated by $\Omega_{0}$ is about 1.5 times the initial orbital period, decreasing slightly at a rate comparable to the rate at which the 


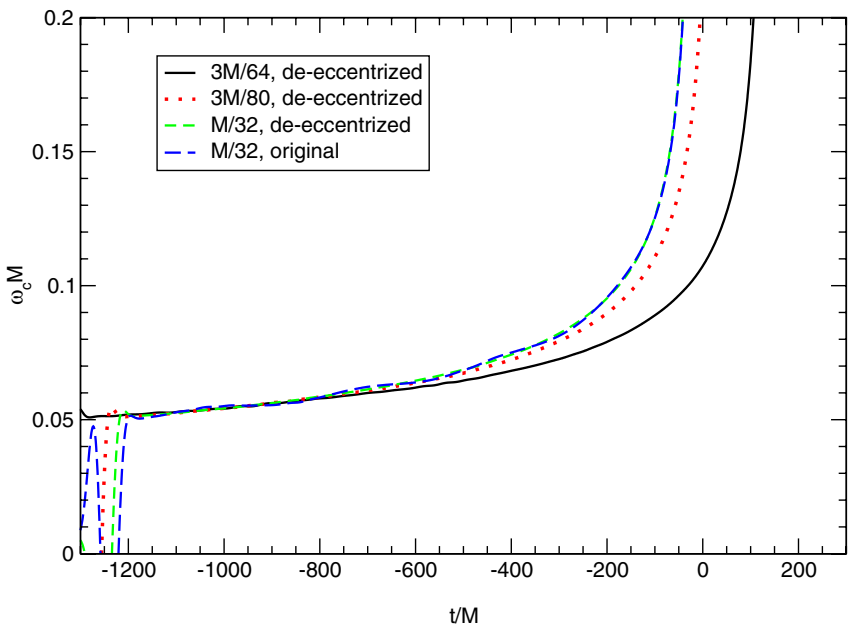

FIG. 7 (color online). Angular frequencies with eccentricity removed, aligned such that the frequencies agree when the $h_{f}=$ $M / 32$ simulation is $1000 M$ before the peak in $\psi_{4}$. Also shown is the frequency from the $h_{f}=M / 32$ resolution before the eccentricity was removed.

wave period grows. Allowing $A$ to evolve in time did not result in clearly improved fits.

From these fits we define eccentricity based on the effect, in Keplerian dynamics, of small eccentricity on orbital frequency, which should provide a good approximation in the adiabatic regime. Kepler's second law (conservation of angular momentum) implies that $L \propto r^{2} \omega$ is constant, from which it follows that the eccentric frequency deviation $d \omega / \omega$ is twice the eccentric radial deviation $d r / r$, suggesting the unitless definition of eccentricity $e \equiv A / 2 \omega_{c}$. Note that, to second order in $e$, this definition of eccentricity is also equivalent to that put forth in Ref. [37] in a post-Newtonian context. The constancy of $A$ in our fit is consistent with a linear decrease in eccentricity as frequency increases. The initial eccentricity $e_{0}$, obtained in this way, is also given in Table II.

Even more interesting than the estimates for eccentricity provided this way are the residual curves $\omega_{c}(t)=\omega(t)-$ $d \omega(t)$ of angular frequency vs time with the eccentric part subtracted out. Note that the strictly sinusoidal nature of our fit to the eccentric modulations represented by $d \omega(t)$ implies that the underlying secular trend should be preserved. Figure 7 shows the results for each of our three simulations together with the unmodified frequency $\omega(t)$ for the high-resolution case. The plotted curves have also

TABLE II. Values resulting from eccentricity fitting. The magnitude of the eccentricity in these simulations (as given by $A M$ or $e_{0}$ ) appears to be at least second-order convergent.

\begin{tabular}{l|lcccc}
\hline \hline Run & $A M$ & $\Omega_{0} M$ & $\dot{\Omega} M^{2}$ & $\Phi_{0}$ & $e_{0}$ \\
\hline $3 M / 64$ & $5 \times 10^{-4}$ & 0.017 & $3 \times 10^{-6}$ & 1 & 0.005 \\
$3 M / 80$ & $7 \times 10^{-4}$ & 0.016 & $4 \times 10^{-6}$ & 1 & 0.007 \\
$M / 32$ & $8 \times 10^{-4}$ & 0.017 & $3 \times 10^{-6}$ & 1 & 0.008 \\
\hline \hline
\end{tabular}

been filtered to remove some high-frequency simulation noise present in the early part of the simulations. The smooth, now monotonic, trend in the curves for $\omega_{c}(t)$ is an indication that most of the wiggles evident in $\omega(t)$ were consistent with our model for eccentric modulations $d \omega(t)$, though the early part is affected by transient features related to the shortcomings of the initial-data model.

We can take $\omega_{c}(t)$ as providing a record of the "hardening" process, as radiative losses bring the system through tightening orbits. The key effect of numerical simulation error evident in Fig. 7 is a slowing of the hardening rate at low resolutions, causing the final merger to be delayed. This delay appears to converge at fourth order in resolution. Viewing the eccentric modulation as a small perturbation on the dynamics of an optimally noneccentric inspiral, we expect that these trends would also provide a good approximation for the frequency evolution of a merger simulation begun with optimally noneccentric initial data.

\section{Waveform accuracy}

In this section we consider the accuracy of the waveforms generated by our simulations, focusing primarily on the accuracy of waveform phasing information in the lateinspiral portion of our simulations. Over the course of many developmental simulations leading up to these results, we found that this early low-frequency part of the simulations is the most difficult to simulate accurately. This makes sense because the crucial dynamical details are in the slow loss of energy and angular momentum to the relatively fine, evolving structure of the spacetime curvature, which ultimately comprises the radiation. Time scales are also longer for this part of the dynamics, requiring high accuracy over a large number of computational iterations.

Figure 8 compares the $\psi_{4}$ waveforms generated by our simulations at different resolutions.

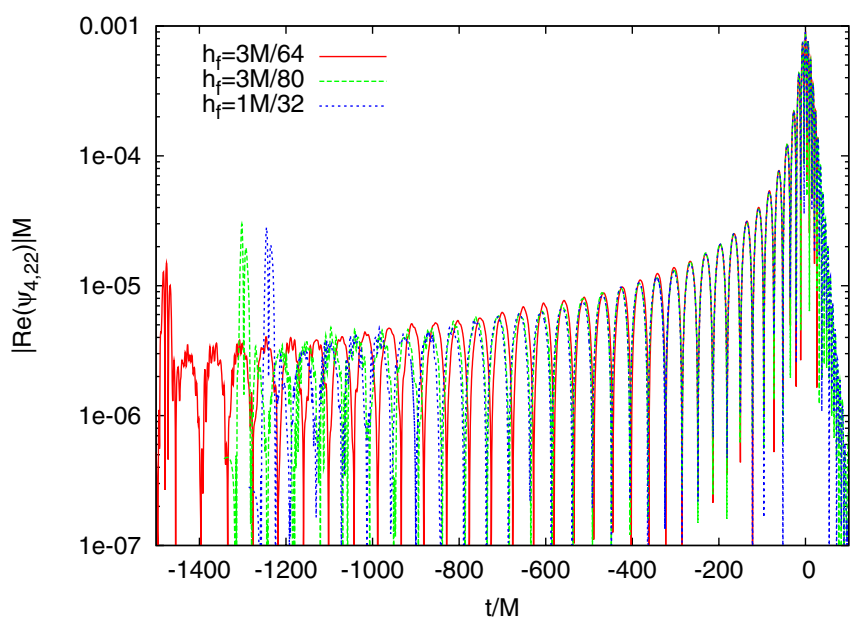

FIG. 8 (color online). Waveform at three resolutions. They have been shifted in time and phase to agree at the peak of the wave. The agreement persists backwards in time, with the growing discrepancy in phase converging away with resolution. 
These waveforms have been aligned to coincide at the peak in $\psi_{4}$ for each of the simulations, as described in Ref. [15]. We show the waveform logarithmically because the amplitude changes by more than 2 orders of magnitude through a run, part of what makes these simulations challenging. We are especially interested in the phase agreement among the different simulations, evident in the nearly vertical parts of the curves (which approach zero crossings). Aligned in this way, waveforms generated at all three resolutions are nearly exactly superposed after $t \sim$ $-250 M$. The two higher-resolution simulations are nearly identical after $t \sim-500 \mathrm{M}$ and agree to within a small part of a cycle for the full portion of the simulation after the initial transient period in the first $100 \mathrm{M}$ of each run. On the other hand, the waveforms are easily distinguished by large differences in the overall timing of each run, with the lowest-resolution simulation going through a full additional orbit before merger. The high-frequency noise evident in the first part of the simulations seems to be caused by reflections from our refinement boundary interfaces.

We get a more direct view of phasing information by examining the waveforms in polar decomposition. In Fig. 9 we show the polarization phase derived from the strain rate waveform. This time we have aligned the simulations in time from the beginning, as should be appropriate for simulations of the same initial configuration. Later in the simulations, as the frequency increases the phase increases more rapidly, and the timing differences among the simulations lead to large phase differences.

We quantitatively compare the phasing results in Fig. 10, showing the difference between the two higher-resolution runs compared with scaled versions of the difference between the two lower-resolution runs. We can now clearly see that the phase errors (measured this way) grow strongly in time. The lower-resolution difference has been rescaled

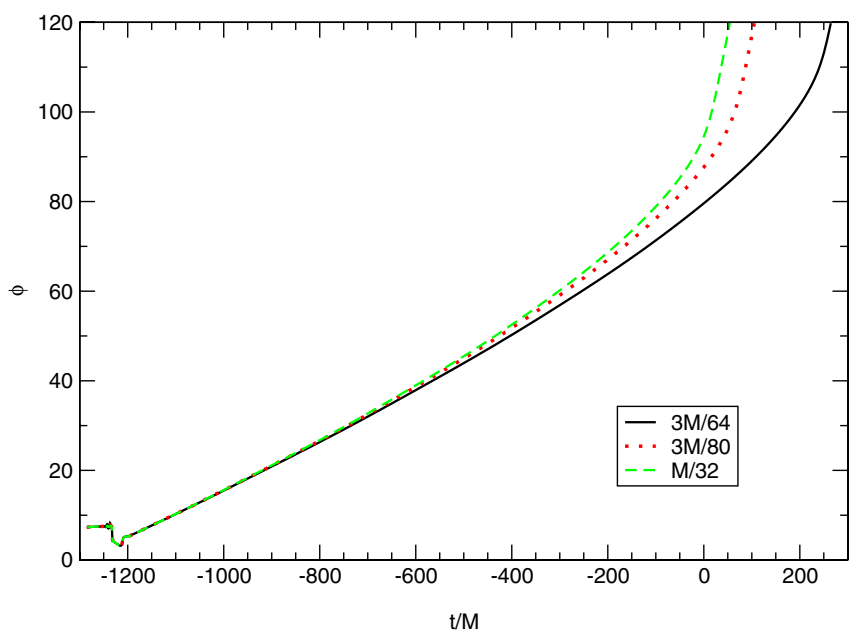

FIG. 9 (color online). Strain rate phase. The high-resolution simulation goes through about 14 cycles before merger. The lower-resolution simulations take longer to merge, and go through more cycles.

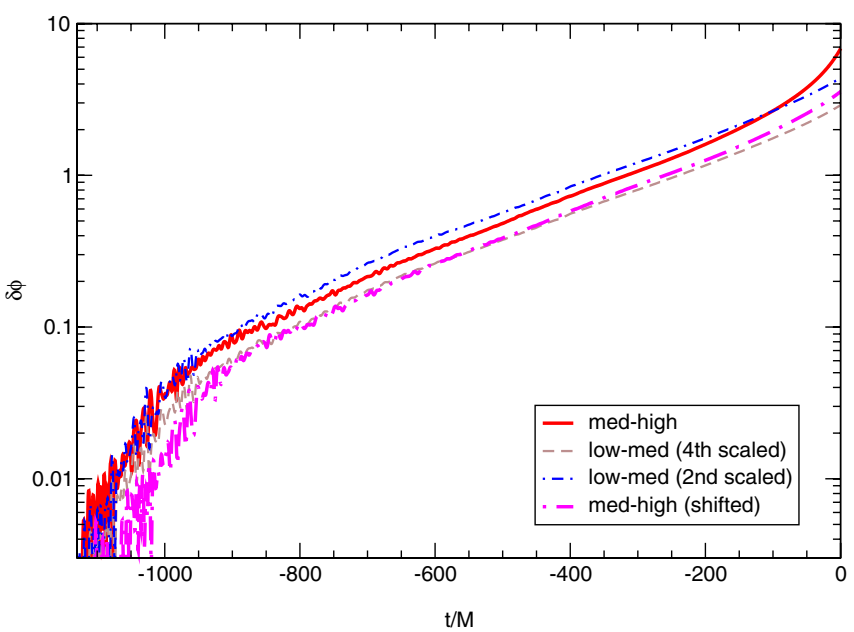

FIG. 10 (color online). Strain rate phase three-point convergence. The higher-resolution phase difference is shown with and without a phase shift to allow comparison of errors at similar dynamical points in the simulation. After shifting, the phaseerror appears to be fourth-order convergent for much of the simulation.

two ways, such that they would be expected to agree with the higher-resolution difference for second- and fourthorder convergence, respectively. The comparison suggests phasing convergence somewhere between second- and fourth-order over much of the run.

Since the phase error grows strongly in time, it is interesting to consider the effect of timing errors in these convergence comparisons. The last curve in the plot shows the high-medium difference shifted in time by $57 M$; that is, the high resolution and medium resolution results have been differenced first, with their original time-dependence unaltered, and then the resulting difference has been shifted in time. $57 M$ represents the approximate timing difference between the two higher-resolution runs late in the simulation, as measured from the peaks in $\psi_{4}$. Viewed this way we see time-aligned phase differences taken from similar points in the physical evolution of the medium resolution run, as represented in the medium-low curve, and the high-resolution run, as represented in the highmedium curve. As a result, the runs appear to converge at a faster rate, closer to fourth order. This unconventionally time-shifted plot is not intended to serve as a rigorous assessment of convergence, but is intended to illustrate that the timing differences can have a significant impact on convergence estimates.

Above, we have compared our simulations made at different resolutions by comparing the waveforms at equal points in time, with time aligned either at the beginning of the simulation ( $t=0$ in the original run) or at the peak in $\psi_{4}$. Since errors cause the simulations to accelerate through their dynamical processes at somewhat different rates, such comparisons end up relating moments of quite different dynamics, and become less meaningful, depending signifi- 
cantly on the reference time according to which the phases are compared. This sort of comparison would not be applicable at all when there is no clear way to physically align predictions in time, such as when comparing numerical simulations with PN results. We can avoid assigning a reference time by using the gravitational wave frequency as the reference.

For the case studied here, a quasicircular inspiral of comparable-mass black holes, it is appropriate to consider the waveform frequency $\omega=\partial \phi / \partial t$ as a gradually increasing monotonic function of time, $\omega \equiv$ $\omega(t)$. Though the actual waveform frequency of our simulations is not monotonic because of small eccentricities in the inspiral trajectories, we have shown that we can fit out the eccentric deviations. The residual secular trend $\omega_{c}(t)$ provides a monotonic frequency, which we can apply as an independent variable against which to compare various simulations.

We show frequency-based phase differences among the simulations in Fig. 11, which allows us to compare the difference between the two higher-resolution simulations with that between the two lower-resolution simulations. If the simulation errors are fourth-order convergent, then the low-medium difference should be approximately 2.784 times the medium-high difference. As is evident from the medium-high " $(3 M / 80-M / 32) "$ and lowmedium " $(3 M / 64-3 M / 80) / 2.784$ " curves in Fig. 11, the errors appear slightly overconvergent with respect to fourth-order scaling. This may be caused by phase error accrued early in the lowest-resolution $\left(h_{f}=3 M / 64\right)$ simulation, due to difficulty in resolving high-frequency components in the spurious Bowen-York radiation, as well as in an initial gauge pulse, which dominate at this time. This lowest resolution may therefore not quite be in the convergent regime during this early part of the simulation. If the phases are adjusted by a constant such that they match at some point after the main part of the BowenYork pulse has passed, then fourth-order scaling fits more closely. This is demonstrated in Fig. 11 by the "( $3 M / 64-3 M / 80) / 2.784$ (shifted)" curve, which has been vertically phase-shifted by a constant so as to agree with the " $(3 M / 80-M / 32)$ " curve at $\omega m_{f}=0.05423$, the frequency $1000 M$ before merger in our high-resolution simulation.

As is also clear in the figure, phase error accumulates most significantly in the low-frequency portion of the simulation, $m_{f} \omega \lesssim 0.08$. This makes sense generally since the simulation spends much more time at lower frequencies. Consider, for instance, the effect of a small nonconservative leakage of energy $\delta \dot{E}$ from the simulation. When the black holes are well separated, the dynamical development manifested in the sweeping frequency is driven by a slow loss of energy and angular momentum. An energy leakage $\delta \dot{E}$ will change the frequency sweep-rate $\dot{\omega}$ by $\delta \dot{\omega} \sim(\delta \dot{E}) /(\partial E / \partial \omega)$, where $\partial E / \partial \omega$ indicates how the

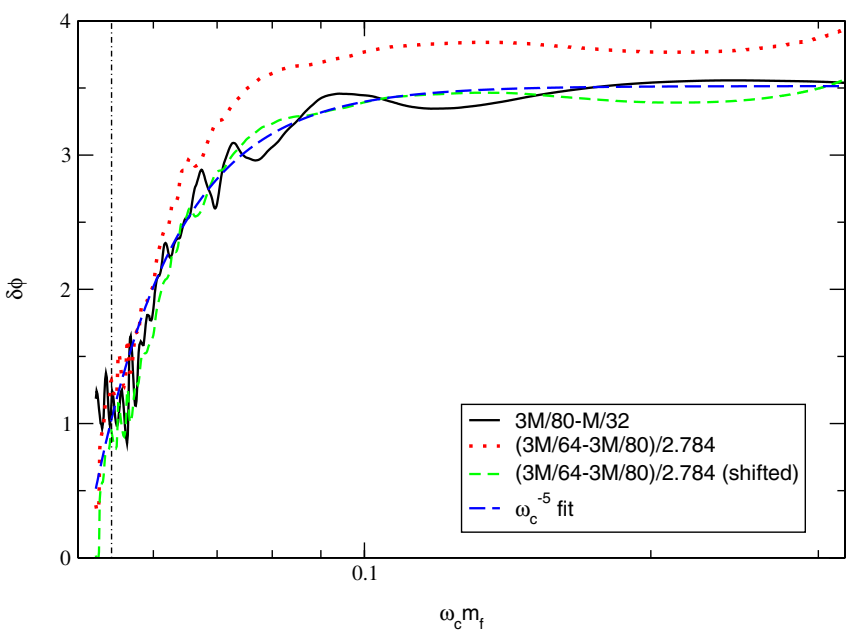

FIG. 11 (color online). Frequency-based phase comparisons for runs at three resolutions. The solid curve represents the phase-difference between the $h_{f}=3 M / 80$ and $h_{f}=M / 32$ simulations; the dotted curve represents the phase-difference between the $h_{f}=3 M / 64$ and $h_{f}=3 M / 80$ simulations; the short-dashed curve represents the phase-difference between the $h_{f}=3 M / 64$ and $h_{f}=3 M / 80$ simulations, shifted vertically by a constant phase so as to agree with the higher-resolution difference at $\omega_{c} m_{f}=0.05423$ (shown as vertical dot-dashed line); and the long-dashed curve shows a fit to $\omega^{-5}$ phasing error, which might be expected if there are energy conservation violations that are constant in time. The lower-resolution curves have been scaled so that they should superpose with the higherresolution curves in the case of fourth-order convergence. The unshifted phase-difference curves appear better than fourth-order convergent, while shifting the lower-resolution curve makes the rate of convergence look closer to fourth order.

binding energy changes with frequency. Phase error $\delta \phi$ can be determined from the error in the sweep-rate by

$$
\delta \phi=\delta \int \frac{\omega}{\dot{\omega}} \mathrm{d} \omega=-\int \frac{\omega}{\dot{\omega}^{2}} \delta \dot{\omega} \mathrm{d} \omega .
$$

Applying the leading-PN-order expressions for $\partial E / \partial \omega$ and $\dot{\omega}(\omega)$ gives a result proportional to $\omega^{-5} \delta \dot{E}$. Indeed this dependence fits our phase differences rather well, as shown in Fig. 11. Note, however, that this does not single out energy nonconservation as the source of error, as other errors may produce similar effects. A similar leakage of angular momentum would lead to error proportional to $\omega^{-4}$, which also fits reasonably well.

The convergence evidenced by Fig. 11 suggests that we can apply Richardson extrapolation to estimate the difference of our high-resolution $(M / 32)$ run from the infiniteresolution limit. If we assumed the fourth-order convergence suggested by Fig. 11, the phase-error estimate for this run would be 0.93 times the difference between the phases from the $3 M / 80$ and $M / 32$ resolution runs. However we will simply take the more conservative estimate of the actual difference between these resolutions. Note that during the last $1000 M$ of our $M / 32$ simulation 
(i.e. from $\omega m_{f}=0.05423$ onwards), we estimate roughly two and a half radians of phase error accumulate, as measured with respect to frequency, which is less than half of a gravitational wave cycle. A benchmark for accumulated waveform phasing errors is one-half of a cycle, because phase error exceeding this amount would lead to destructive interference in matched-filtering applications. For our high-resolution simulation, we estimate less than one-half cycle of gravitational wave phase error over the full simulated waveform, excluding the meaningless transients in the first $100 M$. As in [23], we estimate that these phasing errors are smaller than the implicit phasing difference between the 3PN and 3.5PN expansions of $\dot{\omega}(\omega)$ after $t \sim-300 M\left(\omega_{c} M \sim 0.08\right)$. For our data analysis considerations we will only be using the numerical waveform after this point, for which the estimated phase error is well below a half cycle.

Frequency-based phase comparisons, such as we have presented here, are better suited than time-based phase differences, which depend strongly on where the waveforms are chosen to be aligned in time. The relationship between the two can be understood by considering a oneparameter family of waveform results, with a parameter $\lambda$ representing model dependence, in this case the numerical grid spacing. The waveforms would provide phase as a function of time $\phi_{\lambda}(t)$, from which we can derive frequency $\omega_{\lambda}(t)$, which is monotonic for small eccentricity. Inverting to obtain $t_{\lambda}(\omega)$ one can derive the frequencybased phasing $\bar{\phi}_{\lambda}(\omega) \equiv \phi_{\lambda}\left(t_{\lambda}(\omega)\right)$. Now considering variations $\delta \equiv d / d \lambda$ near $\lambda=0$, one finds the relationship between frequency- and time-based phase comparisons

$$
\delta \bar{\phi}(\omega)=\delta \phi(t(\omega))+\omega \delta t(\omega) .
$$

This sheds some light on the often confusing issue of time alignment in the time-based comparisons shown earlier. Specifically, for a waveform that sweeps significantly through frequency, time- and frequency-based phase differences will be most similar when the time-based phase differences are aligned so that $\delta t$ vanishes where $\omega$ is largest. In our case, the net frequency-based phase differences in Fig. 11 are closer to net phase differences with time aligned at the end of the waveform, as in Fig. 8, than when time is aligned at the lower-frequency beginning, as in Figs. 9 and 10.

\section{APPLICATIONS TO GRAVITATIONAL WAVE OBSERVATIONS OF BBHS}

In the first part of this paper, we presented a state-of-theart calculation of the late inspiral and merger of equal mass, nonspinning BBHs, starting $\sim 7$ orbits before the peak radiation amplitude. In Ref. [23] we showed that there is a significant region over which the waveform from the $M / 32$ numerical simulation presented above agrees with waveforms derived from PN calculations. In this section we will use the best of both treatments by joining the final segment of the BBH evolution with the PN approximation for the preceding inspiral, as shown in Fig. 12. We will show below that the phasing information for this waveform has an estimated accuracy of better than one-half of a gravitational wave cycle, making the waveform applicable in a variety of matched-filtering-based gravitational wave data analysis applications. We use this waveform to calculate the relative detectability of the inspiral and the merger-ringdown, as well as the detectability of the entire waveform, for iLIGO, adLIGO, and LISA. We show examples of characteristic signal strains for both classes of detectors. We also compute SNRs for astrophysically interesting BBH masses, highlighting the importance of the late inspiral and merger parts of the signal. Modifications of our results that may arise from BBHs with spins and unequal masses are considered.

\section{A. Waveform matching and SNR calculation}

The PN waveform used for all the analyses in this section is of order 3.5PN in phase (as derived in [38,39]) and 2.5PN beyond the quadrupole approximation in amplitude (as derived in [40]). It should be noted that the PN approximation that we use for the phase is actually an expansion of the chirp rate $\dot{\omega}$ in terms of the frequency $\omega$, which we then numerically integrate. Direct numerical integration of the 3.5PN expansion of the chirp rate, $\dot{\omega}_{3.5 \mathrm{PN}}$, for example, in the integrand $d \omega\left(\omega / \dot{\omega}_{3.5 \mathrm{PN}}\right)$, does not strictly respect the PN approximation of the phase, as the latter would require additional 3.5PN expansion of the integrand itself. However, the phase obtained in this manner will have the same convergence properties as the original $\dot{\omega}_{3.5 \mathrm{PN}}$ expansion, which is arguably a more fundamental PN quantity because of its close relationship to the rate of energy loss, $\dot{E}$, from which it is derived. Additional expansion of the phase appears to compromise its accuracy. Using shorter runs it had been observed in [21] that the phase obtained from numerical integration of the PN expansion of the chirp rate seemed to agree better with numerical simulations than did the analytic expressions; more recently it was demonstrated in [23], using runs extending well into the late inspiral and with the effects of eccentricity mitigated, that the numerically integrated phase appears to be converging to the numerical result at frequencies where the full 3.5PN expansion of the phase is clearly invalid. We therefore use the numerically integrated 3.5PN chirp rate for the phasing of the PN portion of our waveform.

Figure 12 shows our numerical waveform from Sec. II overlaid with the PN waveform that was just described. To generate a complete, mass-scalable waveform, we match the frequency of the numerical simulation to the PN prediction, adjusting the phases to also be equal at that point as shown in Fig. 12, and connecting the two halves to make a single waveform. This is done by shifting the PN waveform until the frequency equals the numerically predicted fre- 


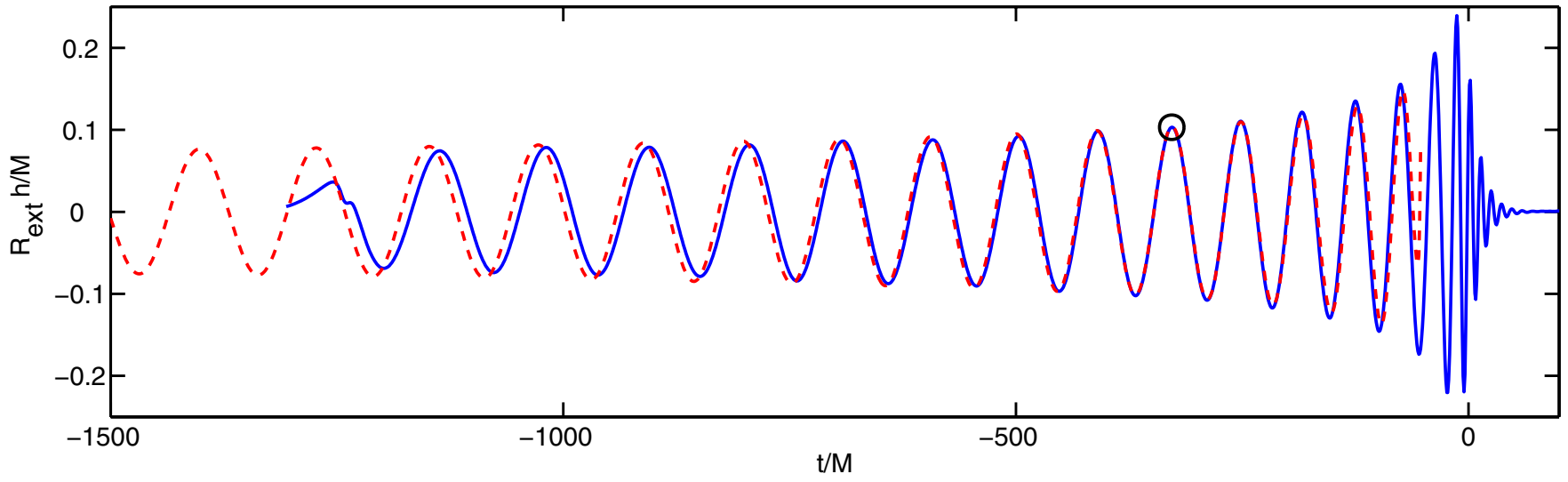

FIG. 12 (color online). Numerical simulation results for gravitational wave strain compared with post-Newtonian estimates. The waveform shown is from the high-resolution numerical simulation presented in Sec. II overlaid here with a PN waveform with 3.5PN order phasing and 2.5PN order amplitude accuracy. The combined waveform, joined at $t=-328 M$ (circle) is applied in Sec. IV to calculate signal-to-noise ratios for iLIGO, adLIGO, and LISA.

quency at a time in the simulation where the accuracy of the numerical data first surpasses the accuracy of the PN approximation, as estimated in [23]. Specifically, [23] predicts this point of equivalent accuracy to occur at $M \omega \sim 0.08$, which corresponds to $t=-328 M$ (shown by the circle in Fig. 12). It is worth noting that there was no need to adjust the PN amplitude for continuity. The amplitude agreement with the numerical simulation is so good, and hence the resulting amplitude is so nearly continuous, that the small discontinuity fails to produce any discernible artifacts in the Fourier transform $\tilde{h}(f)$ of the resulting waveform.

Having generated a waveform, it is informative to estimate the waveform's phasing accuracy over the course of the BBH evolution. Note in Fig. 11 that for the portion of our $M / 32$ simulation that is used in the waveform, we estimate $\sim 0.5 \mathrm{rad}$ of phase error. If we take the difference between 3 and 3.5PN terms to be an estimate of the phase error as in [23], we can assess the error for the PN portion of the waveform. It was shown in [41] that the analytic PN phase expression accumulates very little error, on the order of 0.1 radians, until $M \omega \sim 1 \times 10^{-4}$. Beginning our numerical phase integration at this point and evaluating up to $M \omega=0.08$ yields a gravitational wave phase error of $\sim 3.6 \mathrm{rad}$, such that the total accumulated phase error over the entire waveform is $\sim 4 \mathrm{rad}$. As stated previously, an accumulated waveform phasing error of less than $\pi$ rad is the threshold below which wave-matching comparisons may be used for matched-filtering applications. We estimate that our combined waveform meets this criterion after a frequency of about $M \omega \sim 0.01$ up to the ringdown frequency, $M \omega \sim 0.5$. We therefore have a waveform with sufficient accuracy to be useful as a template for gravitational wave detection. While templates will ultimately be needed for cases of greater astrophysical interest, and still greater accuracy will be required for the template to be useful for the purpose of parameter estima- tion, the construction of this waveform illustrates that the field of numerical relativity has matured to the point of being capable of producing results that are useful for gravitational wave data analysis.

The calculated waveform that we have just described is actually the total strain on the equatorial plane, where $h_{\times}$ vanishes and therefore $h_{+}$provides the only contribution. To get the optimally oriented strain amplitude (which is the total strain passing an observer on the equatorial axis), we multiply this result by $2 \sqrt{2}$, which is the ratio of peak total gravitational wave amplitude to the amplitude of $h_{+}$alone in the quadrupole approximation. We then simply divide by $\sqrt{5}$ in order to convert to an orientation-averaged waveform for our subsequent analyses. This factor can be understood by observing that orientation averaging is fully equivalent to averaging over all sky positions of the detector from the perspective of the BBH, and such sky averaging results in a factor $1 / \sqrt{5}$ in sensitivity [1].

The SNR is calculated assuming matched filtering is performed on the data, and that the waveforms are perfect copies of the embedded signal. In this case, the sky- and waveform-polarization-averaged SNR is given by

$$
\left\langle(\mathrm{SNR})^{2}\right\rangle=\int d(\ln f)\left(\frac{h_{\text {char }}(f)}{h_{n}(f)}\right)^{2},
$$

where $h_{\text {char }}(f) \equiv 2 f|\tilde{h}(f)|$ is the characteristic signal strain and $h_{n}(f) \equiv \sqrt{5} h_{\mathrm{rms}}(f)=\sqrt{5 f S_{n}(f)}$ is the rms of the detector noise fluctuations multiplied by $\sqrt{5}$ for sky averaging, with $\tilde{h}(f)$ and $S_{n}(f)$ being the Fourier transform of the signal strain and the power spectral density of the detector noise, respectively [1].

The waveform scales with luminosity distance $D_{L}$ and total mass $M$ as $h_{\text {char }} \propto(1+z) M / D_{L}$, while the time axis for an observed wave, after redshifting, scales as $t \propto(1+$ $z) M$, so that the waveform shown in Fig. 12 is applicable over all total masses and redshifts. When needed we relate 
luminosity distance to redshift $z$ using cosmological parameters consistent with the most recent Wilkinson Microwave Anisotropy Probe (WMAP) results $\left(\Omega_{\Lambda}=1-\right.$ $\Omega_{M}=0.72, h=0.73$ ) [42] and the relation

$$
D_{L}(z)=\frac{(1+z) c}{H_{0}} \int_{0}^{z} \frac{d z^{\prime}}{\sqrt{\Omega_{M}\left(1+z^{\prime}\right)^{3}+\Omega_{\Lambda}}} .
$$

For cases where an impractically long time series would be needed to cover the band with an adequate sampling rate, the waveform is extended in Fourier space to still lower frequencies (and consequently back further in time) using the quadrupole formula,

$$
\left|\tilde{h}_{\text {quad }}(f)\right|=\frac{1}{2 \sqrt{15} D_{L}}\left(\frac{[(1+z) M]^{5}}{\pi^{4} f^{7}}\right)^{1 / 6} .
$$

The PN portion of the waveform continues slightly past where its Fourier transform deviates from the quadrupole expression for $|\tilde{h}(f)|$ by $\sim 2 \%$, at which point (5) is used to extend $|\tilde{h}(f)|$ back as far as necessary. The PN segment is truncated at a higher frequency than the lowest frequency component of its Fourier transform in order to eliminate edge effects. Finally, the quasinormal ringdown at the end of the numerical simulation is extended by fitting a damping coefficient and fundamental frequency to the data in order to mitigate edge effects at the high-frequency end of the Fourier transform.

\section{B. Observing stellar BBHs and intermediate mass bi- nary black holes (IMBBHs) with iLIGO and adLIGO}

Ground-based interferometers are sensitive to relatively high-frequency gravitational waves from coalescing stellar mass $\left(M \lesssim 10^{2} M_{\odot}\right)$ and intermediate mass (IM) $\left(10^{2} M_{\odot} \lesssim M \lesssim 10^{3} M_{\odot}\right)$ BBHs. In this section, we apply our combined waveform to consider the response of iLIGO and adLIGO to BBH coalescence, illustrating the importance of numerical simulation results for ground-based detectors. LIGO has facilities in Hanford, WA and Livingston, LA; each facility has an interferometer consisting of two $4 \mathrm{~km}$ long arms, and Hanford also has a $2 \mathrm{~km}$ detector. Both the iLIGO and adLIGO detectors are designed to detect high-frequency gravitational waves, with iLIGO sensitive in the frequency range $40 \mathrm{~Hz} \lesssim f \lesssim$ $8000 \mathrm{~Hz}$ and adLIGO in the range $14 \mathrm{~Hz} \leqslant f \leqslant 10^{3} \mathrm{~Hz}$. Initial LIGO is currently operating at or near the initial design sensitivity in a yearlong scientific data-taking run. Advanced LIGO is a planned upgrade that will increase the detector sensitivity by roughly an order of magnitude across the band. In addition, adLIGO can be tuned to optimize its sensitivity for different sources.

For our analysis of iLIGO, we used the design sensitivity to characterize the detector noise [43]. This sensitivity assumes that the noise is seismically limited below $40 \mathrm{~Hz}$, thermally limited between 40 and $150 \mathrm{~Hz}$, and shot limited above $150 \mathrm{~Hz}$. For adLIGO, unlike iLIGO, we had a choice of tuning configurations. We used the wide-band tuning typically associated with burst sources because of its dramatically superior sensitivity at higher frequencies, where the merger portion from many sources is predicted to occur [44]. This yielded an improved SNR for most masses compared to tunings that were optimized for only the early inspiral portion of the coalescence.

In Fig. 13, we show $h_{\text {char }}$ for several sources plotted relative to the $h_{\text {rms }}$ sensitivity curves for iLIGO (dashed line) and adLIGO (dash-dotted line). We plot these values because the height of $h_{\text {char }}$ above $h_{\text {rms }}$ is an indicator of the SNR, as can be seen by inspecting Eq. (3).

By rescaling we can calculate the SNR as a function of redshifted mass, and particular luminosity distance $D_{L}$. In Fig. 14 we plot the SNR achievable by iLIGO for sources at a luminosity distance $D_{L}=100 \mathrm{Mpc}$ as a function of redshifted mass $(1+z) M$. Here, the dashed line shows the SNR from the early inspiral in the time range $-\infty<$ $t<-1000 M$, which is roughly up to the start of our run. The dotted line shows the SNR for the late inspiral, $-1000 M<t<-50 M$, where $t=-50 M$ is approximately the time at which the merger burst begins. The thin solid line gives the SNR for $-50 M<t<\infty$, and encompasses the merger-ringdown part of the signal. The thick solid line shows the SNR from the entire waveform. Note that the addition of the merger-ringdown waveforms increases the SNR and extends the detectable mass range significantly. The merger-ringdown portion $t>-50 M$ dominates for all equal-mass nonspinning merger observations detectable with SNR larger than 10 at $100 \mathrm{Mpc}$.

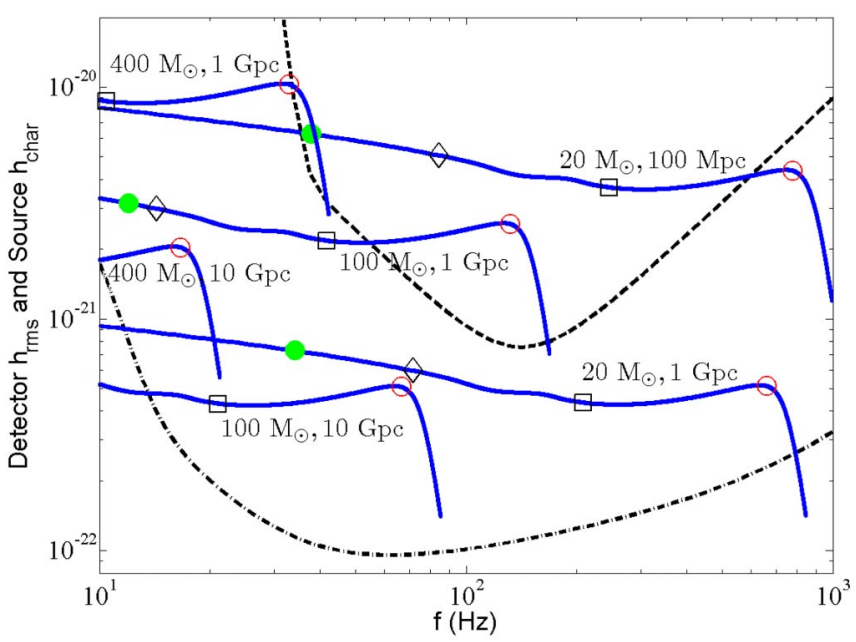

FIG. 13 (color online). The iLIGO (dashed line) and adLIGO (dash-dotted line) rms noise amplitudes $h_{n}$ with the characteristic amplitudes $h_{\text {char }}$ of 6 example sources (solid line). The locations on each $h_{\text {char }}$ corresponding to the peak $\psi_{4}$ amplitude (circle) and $1 \mathrm{~s}$ before the peak in the observer's frame (filled circle), as well as $t=-50 M$ (square) and $t=-1000 M$ (diamond) in the source's frame, are as marked. The mass given is the combined rest mass of each black hole. 


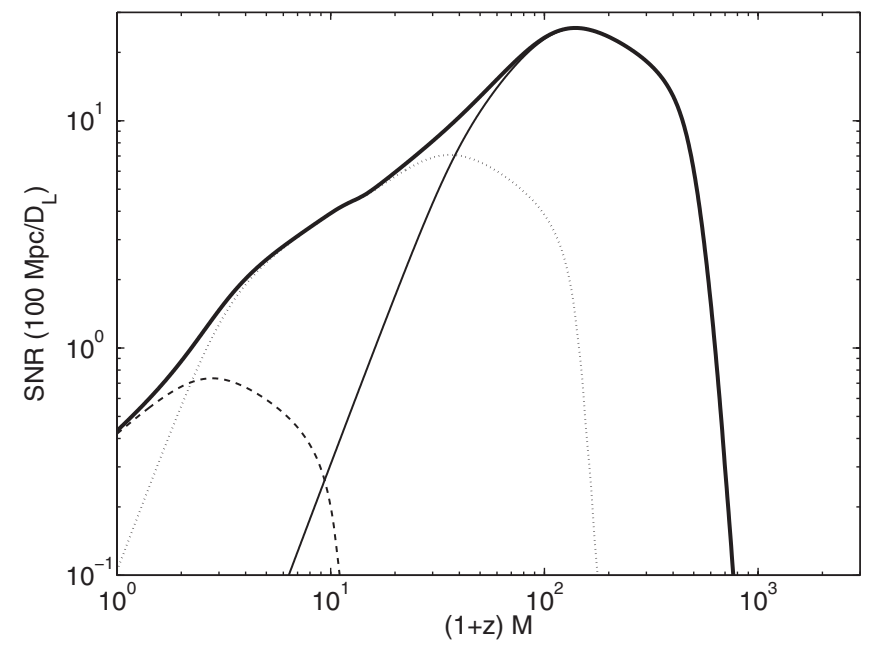

FIG. 14. SNR for sources at luminosity distance $D_{L}=$ $100 \mathrm{Mpc}$ plotted vs redshifted mass for iLIGO. The contributions from $-\infty<t<-1000 M$ (dashed line), $-1000<t<$ $-50 M$ (dotted line), and $-50 M<t<\infty$ (thinner solid line), as well as the SNR from the entire waveform (thicker solid line) are shown.

This type of plot was first made in Ref. [1], and it is useful to compare our results with theirs. Our SNR calculations are based on a full waveform for the case of equalmass, nonspinning black holes. The work in Ref. [1] was done before merger waveforms were calculated and thus is based on estimates for the merger-ringdown regime. For example, they estimated a merger radiation efficiency of $\sim 10 \%$, which is higher than our results but may well obtain for mergers with spin. Comparing their Fig. 4 for the SNR for iLIGO with our Fig. 14 we note that their curve for the inspiral includes the radiation up to the merger and so should be compared to the combination (in quadrature) of our dashed and dotted curves. Our result for the merger SNR is somewhat smaller than theirs, due to the smaller amount of radiation emitted in our mergers. More recently, an analysis of SNR for iLIGO using numerical relativity waveforms for the merger and PN waveforms for the inspiral was made in Ref. [21]; our results in Fig. 14 are similar to what they report in their Fig. 22.

Figure 15 shows the SNR for sources at $D_{L}=1 \mathrm{Gpc}$ for adLIGO. Comparing with Fig. 14, we see that adLIGO will have a significantly higher sensitivity to BBHs over iLIGO. This point is reinforced in Fig. 16, which shows contours of SNR for adLIGO as functions of redshift $z$ and total mass $M$. We find that for $M \sim 200 M_{\odot}$, adLIGO should be able to achieve a SNR greater than 10 out to nearly $z=1$ for equal-mass nonspinning binaries. From Fig. 14 it is evident that these high SNRs depend strongly on the mergerringdown part of the waveform $t>-50 M$.

It is important to note that astrophysical BBHs are likely to have mass ratios different from unity, and that this will reduce the SNRs computed here for the equal-mass case. For stellar BBHs, current work [45] shows that the mass

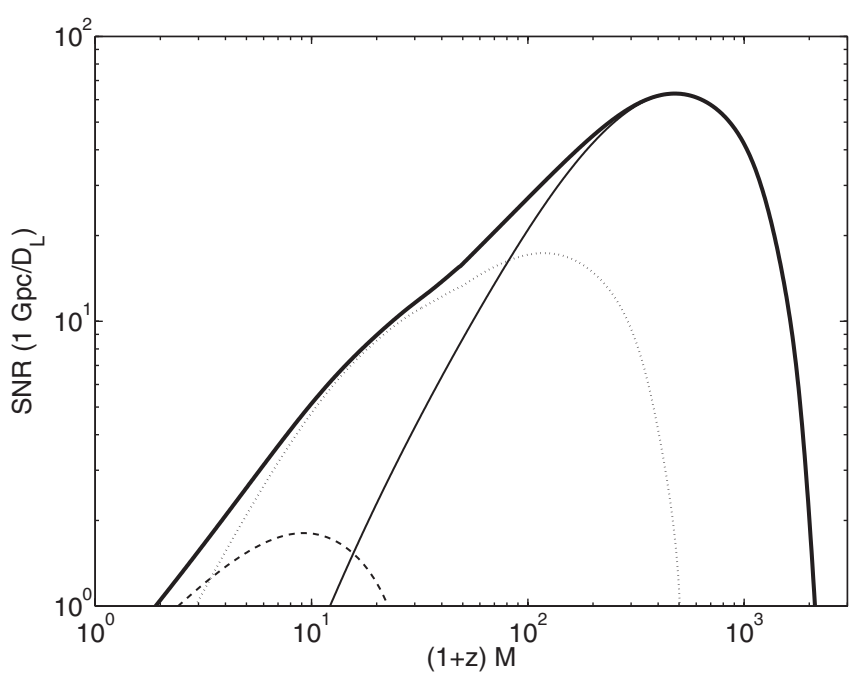

FIG. 15. SNR for sources at luminosity distance $D_{L}=1 \mathrm{Gpc}$ plotted vs redshifted mass for adLIGO. The contributions from $-\infty<t<-1000 M$ (dashed line), $-1000<t<-50 M$ (dotted line), and $-50 M<t<\infty$ (thinner solid line), as well as the SNR from the entire waveform (thicker solid line) are shown.

ratios are rather broadly distributed. The rates for such mergers may be low, $\sim 2 \mathrm{yr}^{-1}$ for adLIGO, depending on the evolution of the original binary through the common envelope phase. For IMBBHs, mass ratios in the range $0.1 \lesssim m_{1} / m_{2} \lesssim 1$ are expected to be the most relevant, with potential rates of $\sim 10$ per year [46], although these rates are far more uncertain than those for stellar BBHs. We can apply the mass scalings from Ref. [1] to show the effect of mass ratios on the computed SNRs; specifically, $\mathrm{SNR} \sim \eta^{1 / 2}$ for the inspiral, and SNR $\sim \eta$ for the merger and ringdown, where $\eta=\mu / M$ and $\mu=m_{1} m_{2} / M$ is the reduced mass. Astrophysical BBHs are also expected to be spinning and this can potentially raise the SNR, for ex-

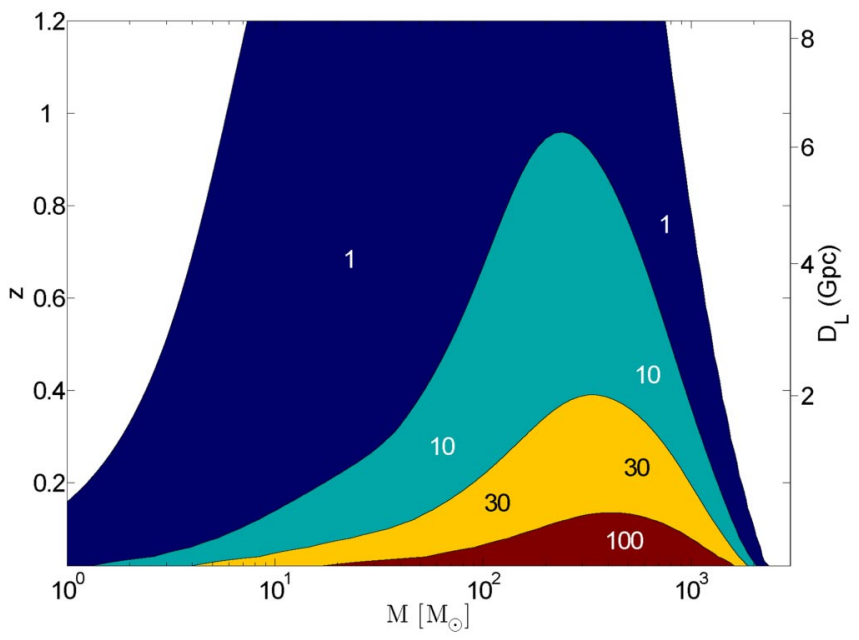

FIG. 16 (color online). SNR contour plot with mass and redshift dependence for adLIGO. 
ample, if there is a spin-induced hangup that generates more gravitational wave cycles in the merger [19].

\section{Observing MBBHs with LISA}

LISA, a proposed space-based interferometer consisting of three $5 \times 10^{6} \mathrm{~km}$ long arms, will be sensitive to lowfrequency gravitational waves from coalescing massive binary black holes (MBBHs) in the band $3 \times 10^{-5} \mathrm{~Hz} \lesssim$ $f \lesssim 1 \mathrm{~Hz}$. The coalescing MBBHs that radiate in this band will have masses $M \gtrsim 10^{4} M_{\odot}$.

Figure 17 shows $h_{\text {char }}$ for several MBBHs plotted relative to the LISA sensitivity curve. We used the "standard" LISA sensitivity curve $[47,48]$ for frequencies above $1 \times$ $10^{-4} \mathrm{~Hz}$, with shot and pointing noise contributions totaling $20 \mathrm{pm} / \sqrt{\mathrm{Hz}}$ of laser phase noise. For $3 \times 10^{-5} \mathrm{~Hz} \leq$ $f \leq 1 \times 10^{-4} \mathrm{~Hz}$, we employed a more conservative estimate of the acceleration noise than the one given in [47], instead assuming a steeper amplitude spectral density that falls off as $f^{-3}$ constrained to match the standard sensitivity curve at $1 \times 10^{-4} \mathrm{~Hz}$ [49]. Below $3 \times 10^{-5} \mathrm{~Hz}$, we assume the detector has no sensitivity, which is a reflection of the uncertainty of the sensitivity at such low frequencies and our desire to make conservative estimates. The sensitivity model assumes that there are no correlated noise

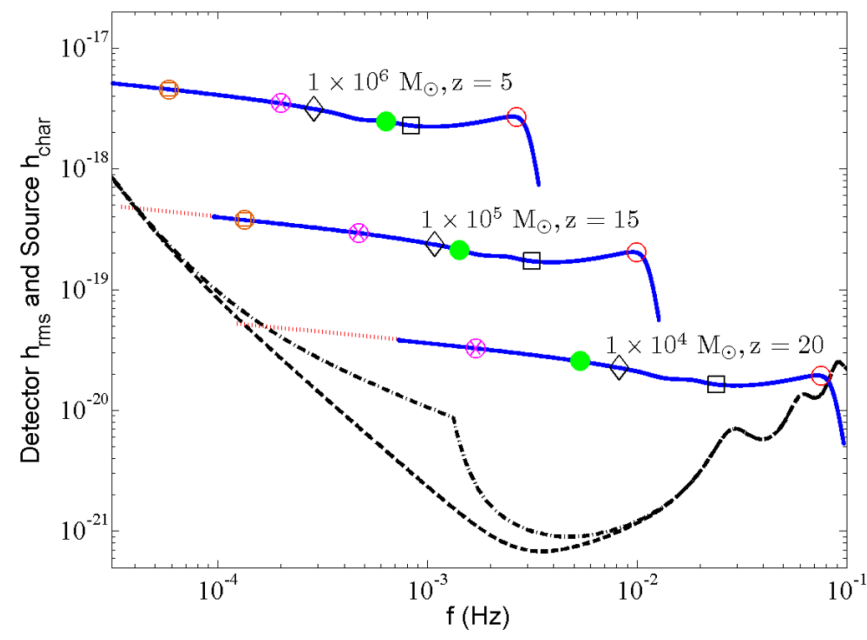

FIG. 17 (color online). LISA rms noise amplitude $h_{\text {rms }}$ from the detector only (dashed line) and from the detector combined with the anticipated white dwarf binary confusion (dash-dotted line) [54] with the characteristic amplitudes $h_{\text {char }}$ of three example sources (solid line). The locations on each $h_{\text {char }}$ curve corresponding to the peak $\psi_{4}$ amplitude (circle), $1 \mathrm{~h}$ before the peak (filled circle), $1 \mathrm{~d}$ before the peak (circle with inscribed cross), and 1 month before the peak (circle with inscribed square) in the observer's frame, as well as $t=-50 M$ (square) and $t=-1000 M$ (diamond) in the source's frame, are as marked. The mass given is the combined rest mass of each black hole. When necessary, the quadrupole approximation is used to extend $h_{\text {char }}$ backward in time 3 years before the peak $\psi_{4}$ amplitude in the detector's frame (dotted line). sources, and its power characterization corresponds to a round trip through one arm of the interferometer.

$\mathrm{MBBH}$ sources can remain in-band for LISA over a very broad frequency range. Therefore, unlike the case of iLIGO and adLIGO, LISA sources nearly always require the use of the quadrupole approximation procedure mentioned above to extend $h_{\text {char }}$ to sufficiently low frequencies. Also, since more massive BBHs chirp more slowly, a MBBH could potentially be in LISA's sensitive band for much longer than the mission's lifetime. To prevent unrealistic SNR values due to this excessive integration time, the quadrupole formula is only used to extend $h_{\text {char }}$ to a low enough frequency such that the total $h_{\text {char }}$ used in our calculations corresponds to 3 years of data in the detector's frame, which is a conservative estimate of the expected mission lifetime.

The SNR for LISA is shown as a function of redshifted mass, normalized for $D_{L}=10 \mathrm{Gpc}$ in Fig. 18. The bump in the curves is caused by the binary confusion noise. Again we see the enhancement of SNR from the mergerringdown part (thin solid line) of the waveforms, and confirm the strikingly large values of SNR obtainable by LISA for these sources seen in [1,21]. For systems with redshifted mass $(1+z) M<3 \times 10^{4}$, the early inspiral $t<$ $-1000 M$ portion of the waveform dominates. The highest SNRs for equal-mass nonspinning mergers are obtained for systems with $(1+z) M>10^{6}$, again dominated by the merger-ringdown portion of the waveform.

Contours of SNR for LISA are shown in Fig. 19 and demonstrate that LISA can observe MBBHs throughout the observable universe at large SNRs. We find it encouraging that, in addition to the large SNR values predicted for LISA

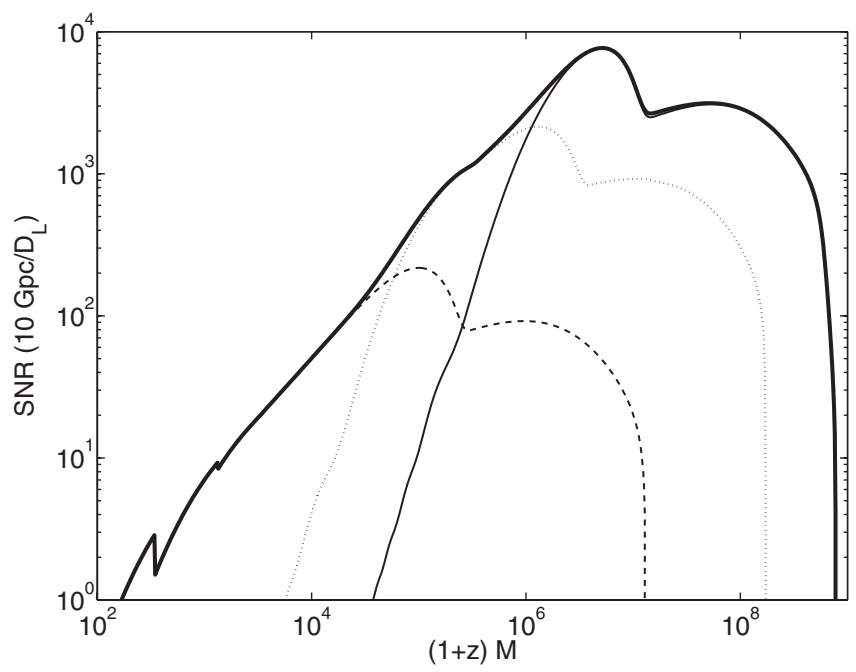

FIG. 18. SNR for sources at luminosity distance $D_{L}=10 \mathrm{Gpc}$ plotted vs redshifted mass for LISA. The contributions from the early inspiral $-\infty<t<-1000 M$ (dashed line), late inspiral $-1000<t<-50 M$ (dotted line), and merger-ringdown $-50 M<t<\infty$ (thinner solid line), as well as the SNR from the entire waveform (thicker solid line) are shown. 


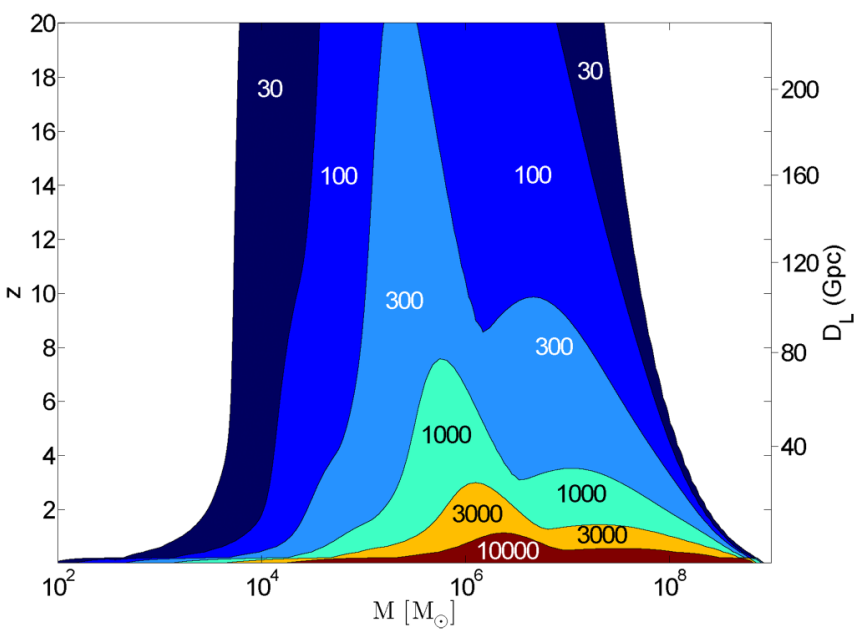

FIG. 19 (color online). SNR contour plot with mass and redshift dependence for LISA. Note that MBBHs with masses $M>$ $10^{7} M_{\odot}$ may not coalesce within a Hubble time [50].

overall, some of the largest values out to the largest redshifts occur in the mass range $10^{5} M_{\odot} \leq M \leq 10^{7} M_{\odot}$ where models of BBH populations predict that the binaries can coalesce within a Hubble time [50] and that the event rates for LISA are several per year [51]. As discussed above, the effects of unequal masses will tend to decrease these SNR values, while spins may increase or decrease them.

Even for nonoptimal configurations, the presence of a $\mathrm{MBBH}$ coalescence in the LISA data stream can dominate

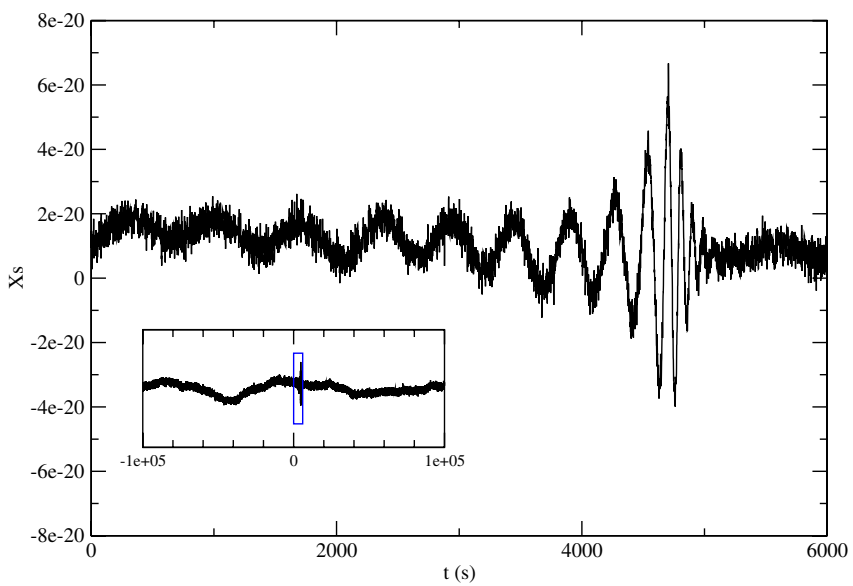

FIG. 20 (color online). Simulated LISA data stream showing LISA's response to a system of two equal-mass black holes $\left(M=10^{5} M_{\odot}\right)$ located at redshift $z=15$ observed on the system's equatorial plane. The quantity plotted is an unequal arm Michelson interferometer observable " $X$ " [55]. The LISA response and instrumental noise are realized using the LISA Simulator [56,57], and colored noise was added to represent the unresolvable galactic binary foreground with the spectrum used in Ref. [54]. The inset shows the signal over a longer duration where low-frequency noise is evident. all the anticipated noise sources. Figure 20 shows a simulation of LISA's response to the merger of equal-mass nonspinning black holes with total mass $M=10^{5} M_{\odot}$ located at redshift $z=15$, and oriented so that LISA lies in the system's equatorial plane, where the radiation is weakest. The SNR for a signal from such a source will be $\sim 200$, averaged over sky positions and polarizations (see Fig. 19).

\section{SUMMARY AND DISCUSSION}

Coalescing BBHs are expected to be the strongest sources for both ground-based interferometers as well as the space-based LISA. In particular, the strong-field merger portion of the gravitational wave signal produces an intense burst of radiation and has the highest luminosity, emitting more energy per second than the combined starlight emitted in the observable universe.

Recent breakthroughs in numerical relativity have opened a new era in understanding the late stages of binary black hole coalescence. We now have a good understanding of the merger-ringdown signal, starting $\sim 50 \mathrm{M}$ before the peak radiation amplitude, for equal-mass nonspinning BBHs. The late-inspiral evolution, that is, more than a few orbits prior to ringdown, is more challenging. Such simulations require better numerical stability and more computational resources, as well as higher accuracy to control the accumulated phase error.

In this paper, we presented new simulations of equalmass nonspinning BBHs starting in the late-inspiral regime and covering approximately an additional factor of 3 in frequency before the merger-ringdown. We carried out runs at three resolutions, $h_{f}=3 M / 64,3 M / 80$, and $M / 32$. Our runs start with relatively low eccentricity and show good convergence and conservation properties. We have demonstrated the stability and accuracy of our simulations over the course of an unprecedented seven orbits. We also showed the value of using frequency (rather than time) to set a reference for the purpose of comparing results between runs as well as with the PN approximation. In recasting phase vs frequency we have found particularly good agreement, not only between the runs but also with PN predictions.

We have also matched the resulting gravitational waveforms to PN calculations covering the earlier parts of the inspiral. The resulting full waveform has less than $3 / 4$ cycle of accumulated phase error over its entire frequency band. Using this waveform, we calculated the SNRs for iLIGO, adLIGO, and LISA. Our results confirm the importance of the merger-ringdown signal, which yields the highest values of SNR for the majority of equal-mass signals $[1,21]$. We also show the SNR for the late-inspiral regime, which numerical simulations are now beginning to address. The late inspiral dominates the SNR for iLIGO and adLIGO for the lower mass ( $\lesssim$ a few $\times 10 M_{\odot}$ ) stellar BBHs, and the SNR for LISA generated by MBBHs with $M \sim 10^{5} M_{\odot}$. Contour plots of 
SNR as a function of $z$ and $M$ show that adLIGO can achieve SNR $\gtrsim 10$ for IMBBHs out to nearly $z \sim 1$, and that LISA can observe MBBHs at SNR $>100$ out to the earliest epochs of structure formation at $z>15$.

Our work has focused on equal-mass nonspinning BBHs. Astrophysically, BBHs are expected to have unequal masses and spins. In general, the effects of unequal masses will tend to decrease the resulting SNRs, while spins can increase them. Calculations of merger-ringdown waveforms for several mass ratios, and for some spins, are currently available; we expect this to be a significant area of focus in the foreseeable future, both expanding the range of parameters studied and extending the duration of the resulting simulations.

As computational technologies mature, simulations of the merger can be used in conjunction with gravitational wave observations to probe gravity in the arena of strong fields. In particular, if the binary masses and spins can be obtained from observations of the inspiral (as demonstrated, e.g., in Ref. [52] for LISA), numerical relativity can be used to calculate the merger waveform. This will allow a comparison between the predictions of general relativity - or indeed, any other theory of gravity used in a numerical simulation - with observations in the regime of very strong gravity.

\section{ACKNOWLEDGMENTS}

We are pleased to acknowledge useful discussions with Chris Belczynski, Scott Hughes, Cole Miller, Peter Shawhan, David Shoemaker, and Tuck Stebbins. This work was supported in part by NASA Grant No. O5BEFS-05-0044. The simulations were carried out using Project Columbia at NASA Ames Research Center and the NASA Center for Computational Sciences at Goddard Space Flight Center. J.v. M., B. K., and M. K. were supported by the NASA Postdoctoral Program at the Oak Ridge Associated Universities. D.C. was supported in part by The Korea Research Foundation and The Korean Federation of Science and Technology Societies Grant funded by the Korean Government (MOEHRD, Basic Research Promotion Fund).
[1] E. E. Flanagan and S. A. Hughes, Phys. Rev. D 57, 4535 (1998).

[2] B. Brügmann, W. Tichy, and N. Jansen, Phys. Rev. Lett. 92, 211101 (2004).

[3] P. Diener et al., Phys. Rev. Lett. 96, 121101 (2006).

[4] S. Brandt and B. Brügmann, Phys. Rev. Lett. 78, 3606 (1997).

[5] F. Pretorius, Phys. Rev. Lett. 95, 121101 (2005).

[6] F. Pretorius, Classical Quantum Gravity 22, 425 (2005).

[7] F. Pretorius, Classical Quantum Gravity 23, S529 (2006).

[8] M. Campanelli, C. O. Lousto, P. Marronetti, and Y. Zlochower, Phys. Rev. Lett. 96, 111101 (2006).

[9] J. G. Baker, J. Centrella, D.-I. Choi, M. Koppitz, and J. van Meter, Phys. Rev. Lett. 96, 111102 (2006).

[10] J. R. van Meter, J. G. Baker, M. Koppitz, and D.-I. Choi, Phys. Rev. D 73, 124011 (2006).

[11] M. Hannam, S. Husa, D. Pollney, B. Brügmann, and N. Ó Murchadha, arXiv:gr-qc/0606099.

[12] B. Brügmann, J. A. Gonzalez, M. Hannam, S. Husa, U. Sperhake, and W. Tichy, arXiv:gr-qc/0610128.

[13] U. Sperhake, arXiv:gr-qc/0606079.

[14] M. Campanelli, C. O. Lousto, and Y. Zlochower, Phys. Rev. D 73, 061501(R) (2006).

[15] J. G. Baker, J. Centrella, D.-I. Choi, M. Koppitz, and J. van Meter, Phys. Rev. D 73, 104002 (2006).

[16] F. Herrmann, D. Shoemaker, and P. Laguna, arXiv:gr-qc/ 0601026.

[17] J. G. Baker, J. Centrella, D.-I. Choi, M. Koppitz, J. van Meter, and M. C. Miller, Astrophys. J. Lett. 653, L93 (2006).

[18] J. A. Gonzalez, U. Sperhake, B. Brügmann, M. Hannam, and S. Husa, Phys. Rev. Lett. 98, 091101 (2007).

[19] M. Campanelli, C. O. Lousto, and Y. Zlochower, Phys. Rev. D 74, 041501(R) (2006).
[20] M. Campanelli, C. O. Lousto, and Y. Zlochower, Phys. Rev. D 74, 084023 (2006).

[21] A. Buonanno, G. B. Cook, and F. Pretorius, arXiv:gr-qc/ 0610122 [Phys. Rev. D (to be published)].

[22] J. Baker, M. Campanelli, F. Pretorius, and Y. Zlochower, Classical Quantum Gravity 24, S25 (2007).

[23] J.G. Baker, J.R. van Meter, S.T. McWilliams, J. Centrella, and B.J. Kelly, arXiv:gr-qc/0612024 [Phys. Rev. Lett. (to be published)].

[24] J. M. Centrella, AIP Conf. Proc. 873, 70 (2006).

[25] A. Buonanno and T. Damour, Phys. Rev. D 59, 084006 (1999).

[26] T. Damour, P. Jaranowski, and G. Schäfer, Phys. Rev. D 62, 084011 (2000).

[27] L. Blanchet, Phys. Rev. D 65, 124009 (2002).

[28] J. D. Brown and L. L. Lowe, J. Comput. Phys. 209, 582 (2005).

[29] W. Tichy and B. Brügmann, Phys. Rev. D 69, 024006 (2004).

[30] P. Hübner, Classical Quantum Gravity 16, 2823 (1999).

[31] M. D. Duez, S. L. Shapiro, and H.-J. Yo, Phys. Rev. D 69, 104016 (2004).

[32] P. MacNeice, K. Olson, C. Mobarry, R. de Fainchtein, and C. Packer, Comput. Phys. Commun. 126, 330 (2000).

[33] http://ct.gsfc.nasa.gov/paramesh/Users_manual/amr.html.

[34] J. Baker, M. Campanelli, and C. O. Lousto, Phys. Rev. D 65, 044001 (2002).

[35] D. R. Fiske, J. G. Baker, J. R. van Meter, D.-I. Choi, and J. M. Centrella, Phys. Rev. D 71, 104036 (2005).

[36] C. W. Misner, Classical Quantum Gravity 21, S243 (2004).

[37] E. Berti, S. Iyer, and C. M. Will, Phys. Rev. D 74, 061503(R) (2006). 
[38] L. Blanchet, G. Faye, B. R. Iyer, and B. Joguet, Phys. Rev. D 65, 061501 (2002); 71, 129902(E) (2005).

[39] L. Blanchet, T. Damour, G. Esposito-Farèse, and B. R. Iyer, Phys. Rev. Lett. 93, 091101 (2004).

[40] K. G. Arun, L. Blanchet, B. R. Iyer, and M. S. S. Qusailah, Classical Quantum Gravity 21, 3771 (2004); 22, 3115 (2005).

[41] S. T. McWilliams and J. G. Baker, in Sixth International LISA Symposium, edited by S. Merkowitz and J. Livas (AIP, New York, 2006), p. 110.

[42] D. N. Spergel et al., Astrophys. J. Supp. Ser. 170, 377 (2007).

[43] A. Lazzarini and R. Weiss, LIGO Scientific Collaboration Tech. Rep. No. LIGO-E950018-02 E 25.03.96, 1996, http://www.ligo.caltech.edu/docs/E/E950018-02.pdf.

[44] D. Shoemaker (private communication).

[45] K. Belczynski, V. Kalogera, F. A. Rasio, R. E. Taam, and T. Bulik, Astrophys. J. 662, 504 (2007).

[46] J. M. Fregeau, S.L. Larson, M.C. Miller, R. O'Shaughnessy, and F.A. Rasio, Astrophys. J. 646, L135 (2006).
[47] S. Larson, W. Hiscock, and R. Hellings, Phys. Rev. D 62, 062001 (2000).

[48] S. Larson, http://www.srl.caltech.edu/shane/sensitivity/.

[49] S. M. Merkowitz, in Sixth International LISA Symposium, edited by S. Merkowitz and J. Livas (AIP, New York, 2006), p. 133.

[50] M. Milosavljevic and D. Merritt, Astrophys. J. 596, 860 (2003).

[51] A. Sesana, F. Haardt, P. Madau, and M. Volonteri, Astrophys. J. 623, 23 (2005).

[52] R. N. Lang and S. A. Hughes, Phys. Rev. D 74, 122001 (2006).

[53] C. W. Misner, K. S. Thorne, and J. A. Wheeler, Gravitation (Freeman, San Francisco, 1973).

[54] L. Barack and C. Cutler, Phys. Rev. D 69, 082005 (2004).

[55] D. A. Shaddock, M. Tinto, F. B. Estabrook, and J. W. Armstrong, Phys. Rev. D 68, 061303 (2003).

[56] N. J. Cornish and L. J. Rubbo, Phys. Rev. D 67, 022001 (2003).

[57] N. J. Cornish, L. J. Rubbo, and O. Poujade, The LISA simulator, www.physics.montana.edu/LISA/. 